\title{
On recent advances in the Beltrami equations
}

\author{
Vladimir GutlyanskiĬ, Vladimir Ryazanov, \\ URI SREBro, EduARd Yakubov
}

\begin{abstract}
We give an exposition of the recent progress in the theory of the Beltrami equations with the degeneration.
\end{abstract}

2010 MSC. 30C65, 30C75.

Key words and phrases. Beltrami equations, regular solutions, strong ring solutions, finite mean oscillation.

\section{Introduction}

This paper surveys mainly the recent works of the authors devoted to the degenerate Beltrami equations. The existence problem for the Beltrami equations in the uniformly elliptic case was investigated long ago, see e.g. $[1,8,10]$ and [58]. The existence problem for degenerate Beltrami equations is currently an active area of research. This problem was studied extensively and many contributions have been made, see e.g. [14-16,18,19,21-25,37,38,45-47,50-54,56,57,61,62,67,71,78-87,104,110]. The recent monographs [6] and [63] and the survey [94] make possible for us to restrict ourselves by the discussion basically of our own latest results in the field. We also give a comparison of our theorems with the corresponding earlier contributions.

Our approach for deriving criteria for existence of solutions to the degenerate Beltrami equations is based mainly on the modulus techniques, see the original paper [3], the resent books $[7,26,63,107]$ and the following papers and monographs [6, 49, 55, 92,93,101,111].

In the theory of quasiconformal mappings it is well-known the role of the Beltrami equations of the first type (1.1). Let $D$ be a domain in the complex plane $\mathbb{C}$, i.e., a connected and open subset of $\mathbb{C}$, and let $\mu: D \rightarrow \mathbb{C}$ be a measurable function with $|\mu(z)|<1$ a.e. (almost everywhere) in $D$. The Beltrami equation is the equation of the form

$$
f_{\bar{z}}=\mu(z) \cdot f_{z}
$$

Received 11.11.2010 
where $f_{\bar{z}}=\bar{\partial} f=\left(f_{x}+i f_{y}\right) / 2, f_{z}=\partial f=\left(f_{x}-i f_{y}\right) / 2, z=x+i y$, and $f_{x}$ and $f_{y}$ are partial derivatives of $f$ in $x$ and $y$, correspondingly. The function $\mu$ is called the Beltrami coefficient and

$$
K_{\mu}(z)=\frac{1+|\mu(z)|}{1-|\mu(z)|}
$$

the dilatation quotient for the equation (1.1). The Beltrami equation (1.1) is said to be degenerate if $\operatorname{ess} \sup K_{\mu}(z)=\infty$. The study of the corresponding homeomorphisms started in the frames of the theory of the so-called mean quasiconformal mappings, see e.g. $[2,9,35,36,39,50$, $51,53,54,70,71,76,98,99,105]$.

On the other hand, the Beltrami equations of the second type

$$
f_{\bar{z}}=\nu(z) \cdot \overline{f_{z}}
$$

play a great role in many problems of mathematical physics, see e.g. [52].

The existence problem for the Beltrami equations with two characteristics

$$
f_{\bar{z}}=\mu(z) \cdot f_{z}+\nu(z) \cdot \overline{f_{z}}
$$

where $|\mu(z)|+|\nu(z)|<1$ a.e. was also resolved first in the case of the bounded dilatation quotients

$$
K_{\mu, \nu}(z)=\frac{1+|\mu(z)|+|\nu(z)|}{1-|\mu(z)|-|\nu(z)|},
$$

see Theorem 5.1 in [10]. Recently in [14], the existence of homeomorphic solutions in the Sobolev class $W_{\text {loc }}^{1, s}, s \in[1,2)$, for the equation (1.4) was stated in the case when $K_{\mu, \nu}$ had a majorant $Q$ in the class BMO, bounded mean oscillation by John and Nirenberg, see e.g. [48] and [74]. Note that $L^{\infty} \subset \mathrm{BMO} \subset L_{\mathrm{loc}}^{p}$ for all $p \in[1, \infty)$. Our recent papers [15] and [16] have been devoted to the study of more general cases when $K_{\mu, \nu} \in L_{\text {loc }}^{1}$.

Recall that a function $f: D \rightarrow \mathbb{C}$ is absolutely continuous on lines, abbr. $f \in \mathrm{ACL}$, if, for every closed rectangle $R$ in $D$ whose sides are parallel to the coordinate axis, $f \mid R$ is absolutely continuous on almost all line segments in $R$ which are parallel to the sides of $R$. In particular, $f$ is ACL (possibly modified on a set of Lebesgue measure zero) if it belongs to the class $W_{\text {loc }}^{1,1}$ of locally integrable functions with locally integrable first distributional derivatives and, conversely, if $f \in$ ACL has locally integrable first partial derivatives, then $f \in W_{\text {loc }}^{1,1}$, see e.g. 1.2.4 in [65]. For an orientation-preserving ACL homeomorphism $f: D \rightarrow \mathbb{C}$, the Jacobian $J_{f}(z)=\left|f_{z}\right|^{2}-\left|f_{\bar{z}}\right|^{2}$ is nonnegative a.e. In this case, the complex 
dilatation $\mu_{f}$ of $f$ is the ratio $\mu(z)=f_{\bar{z}} / f_{z}$ if $f_{z} \neq 0$ and $\mu(z)=0$ otherwise, and the point-wise dilatation $K_{f}(z)$ of $f$ is defined in terms of $\mu$ by (1.2). Note that $|\mu(z)| \leq 1$ a.e. and $K_{\mu}(z) \geq 1$ a.e.

Following [15], we call a homeomorphism $f \in W_{\text {loc }}^{1,1}(D)$ a regular solution of (1.4) if $f$ satisfies (1.4) a.e. and $J_{f}(z) \neq 0$ a.e. We also studied the so-called strong ring solutions, see [85-87], that make possible, for instance, to study the Dirichlet problem for the Beltrami equation (1.1), see e.g. $[29,30]$.

\section{BMO, VMO and FMO}

The BMO space was introduced by John and Nirenberg in [48] and soon became one of the main concepts in harmonic analysis, complex analysis, partial differential equations and related areas, see e.g. [41,74].

Let $D$ be a domain in the complex plane $\mathbb{C}$. Recall that a real valued function $\varphi \in \mathrm{L}_{\text {loc }}^{1}(D)$ is said to be of bounded mean oscillation in $D$, abbr. $\varphi \in \mathrm{BMO}(\mathrm{D})$ or simply $\varphi \in \mathrm{BMO}$, if

$$
\|\varphi\|_{*}=\sup _{B \subset D} f_{B}\left|\varphi(z)-\varphi_{B}\right| d m(z)<\infty
$$

where the supremum is taken over all disks $B$ in $D$ and

$$
\varphi_{B}=f_{B} \varphi(z) d m(z)=\frac{1}{|B|} \int_{B} \varphi(z) d m(z)
$$

is the mean value of the function $\varphi$ over $B$. Here and later on $d m(z)$ stands for the Lebesgue measure in $\mathbb{C}$. Note that $\mathrm{L}^{\infty}(\mathrm{D}) \subset \mathrm{BMO}(\mathrm{D}) \subset$ $\mathrm{L}_{\text {loc }}^{p}(\mathrm{D})$ for all $1 \leq p<\infty$, see e.g. [74].

A function $\varphi$ in BMO is said to have vanishing mean oscillation, abbr. $\varphi \in \mathrm{VMO}$, if the supremum in (2.1) taken over all disks $B$ in $D$ with $|B|<\varepsilon$ converges to 0 as $\varepsilon \rightarrow 0$. VMO has been introduced by Sarason in [90]. There is a number of papers devoted to the study of partial differential equations with coefficients of the class VMO, see e.g. $[22,47,64,69,73]$.

Following [44], we say that a function $\varphi: D \rightarrow \mathbb{R}$ has finite mean oscillation at a point $z_{0} \in D$ if

$$
d_{\varphi}\left(z_{0}\right)=\varlimsup_{\varepsilon \rightarrow 0} f_{B\left(z_{0}, \varepsilon\right)}\left|\varphi(z)-\tilde{\varphi}_{\varepsilon}\left(z_{0}\right)\right| d m(z)<\infty
$$

where

$$
\tilde{\varphi}_{\varepsilon}\left(z_{0}\right)=f_{B\left(z_{0}, \varepsilon\right)} \varphi(z) d m(z)
$$


is the mean value of the function $\varphi(z)$ over the disk $B\left(z_{0}, \varepsilon\right)$. Condition (2.3) includes the assumption that $\varphi$ is integrable in some neighborhood of the point $z_{0}$. We call $d_{\varphi}\left(z_{0}\right)$ the dispersion of the function $\varphi$ at the point $z_{0}$. We say also that a function $\varphi: D \rightarrow \mathbb{R}$ is of finite mean oscillation in $D$, abbr. $\varphi \in \mathrm{FMO}(\mathrm{D})$ or simply $\varphi \in \mathrm{FMO}$, if $\varphi$ has a finite dispersion at every point $z_{0} \in D$.

Remark 2.1. Note that, if a function $\varphi: D \rightarrow \mathbb{R}$ is integrable over $B\left(z_{0}, \varepsilon_{0}\right) \subset D$, then

$$
f_{B\left(z_{0}, \varepsilon\right)}\left|\varphi(z)-\tilde{\varphi}_{\varepsilon}\left(z_{0}\right)\right| d m(z) \leq 2 \cdot \tilde{\varphi}_{\varepsilon}\left(z_{0}\right)
$$

and the left hand side in (2.5) is continuous in the parameter $\varepsilon \in\left(0, \varepsilon_{0}\right]$ by the absolute continuity of the indefinite integral. Thus, for every $\delta_{0} \in\left(0, \varepsilon_{0}\right)$,

$$
\sup _{\varepsilon \in\left[\delta_{0}, \varepsilon_{0}\right]} f_{B\left(z_{0}, \varepsilon\right)}\left|\varphi(z)-\tilde{\varphi}_{\varepsilon}\left(z_{0}\right)\right| d m(z)<\infty .
$$

Thus, the condition (2.3) holds if and only if

$$
\sup _{\varepsilon \in\left(0, \varepsilon_{0}\right]} f_{B\left(z_{0}, \varepsilon\right)}\left|\varphi(z)-\tilde{\varphi}_{\varepsilon}\left(z_{0}\right)\right| d m(z)<\infty .
$$

The value of the left hand side of (2.7) is called the maximal dispersion of the function $\varphi$ in the disk $B\left(z_{0}, \varepsilon_{0}\right)$.

Proposition 2.1. If for some collection of numbers $\varphi_{\varepsilon} \in \mathbb{R}, \quad \varepsilon \in\left(0, \varepsilon_{0}\right]$,

$$
\varlimsup_{\varepsilon \rightarrow 0} f_{B\left(z_{0}, \varepsilon\right)}\left|\varphi(z)-\varphi_{\varepsilon}\right| d m(z)<\infty,
$$

then $\varphi$ is of finite mean oscillation at $z_{0}$.

Choosing in Proposition $2.1 \varphi_{\varepsilon} \equiv 0, \varepsilon \in\left(0, \varepsilon_{0}\right]$, we obtain the following statement.

Corollary 2.1. If for a point $z_{0} \in D$

$$
\varlimsup_{\varepsilon \rightarrow 0} \underset{B\left(z_{0}, \varepsilon\right)}{f}|\varphi(z)| d m(z)<\infty,
$$

then $\varphi$ has finite mean oscillation at $z_{0}$. 
Recall that a point $z_{0} \in D$ is called a Lebesgue point of a function $\varphi: D \rightarrow \mathbb{R}$ if $\varphi$ is integrable in a neighborhood of $z_{0}$ and

$$
\lim _{\varepsilon \rightarrow 0} f_{B\left(z_{0}, \varepsilon\right)}\left|\varphi(z)-\varphi\left(z_{0}\right)\right| d m(z)=0 .
$$

It is known that almost every point in $D$ is a Lebesgue point for every function $\varphi \in L^{1}(D)$. We thus have the following corollary.

Corollary 2.2. Every function $\varphi: D \rightarrow \mathbb{R}$, which is locally integrable, has a finite mean oscillation at almost every point in $D$.

Remark 2.2. Note that the function $\varphi(z)=\log (1 /|z|)$ belongs to BMO in the unit disk $\Delta$, see e.g. [74], p. 5, and hence also to FMO. However, $\tilde{\varphi}_{\varepsilon}(0) \rightarrow \infty$ as $\varepsilon \rightarrow 0$, showing that the condition (2.9) is only sufficient but not necessary for a function $\varphi$ to be of finite mean oscillation at $z_{0}$.

The following lemma is a basic tool for applications of the concept of FMO to the existence problem to the Beltrami equations. Below we use the notation

$$
A\left(\varepsilon, \varepsilon_{0}\right)=\left\{z \in \mathbb{C}: \varepsilon<|z|<\varepsilon_{0}\right\}
$$

Lemma 2.1. Let $\varphi: D \rightarrow \mathbb{R}$ be a nonnegative function with finite mean oscillation at $0 \in D$ and let $\varphi$ be integrable in $B\left(0, e^{-1}\right) \subset D$. Then

$$
\int_{A\left(\varepsilon, e^{-1}\right)} \frac{\varphi(z) d m(z)}{\left(|z| \log \frac{1}{|z|}\right)^{2}} \leq C \cdot \log \log \frac{1}{\varepsilon} \quad \forall \varepsilon \in\left(0, e^{-e}\right)
$$

Some versions of this lemma have been first established for BMO functions in [78] and then for FMO functions in [44] and [83].

Clearly that BMO $\subset$ FMO. By definition FMO $\subset L_{\mathrm{loc}}^{1}$ but FMO is not a subset of $L_{\text {loc }}^{p}$ for any $p>1$ in comparison with $\mathrm{BMO}_{\mathrm{loc}} \subset L_{\mathrm{loc}}^{p}$ for all $p \in[1, \infty)$. Here $\mathrm{BMO}_{\mathrm{loc}}$ stands for the local version of the class $\mathrm{BMO}$. So, let us give examples showing that $\mathrm{FMO}$ is not $\mathrm{BMO}_{\mathrm{loc}}$, see [82] and [83], correspondingly.

Example 2.1. Set $z_{n}=2^{-n}, r_{n}=2^{-p n^{2}}, p>1, c_{n}=2^{2 n^{2}}, D_{n}=\{z \in$ $\left.\mathbb{C}:\left|z-z_{n}\right|<r_{n}\right\}$, and

$$
\varphi(z)=\sum_{n=1}^{\infty} c_{n} \chi\left(D_{n}\right)
$$

Evidently by Corollary 2.1 that $\varphi \in F M O(\mathbb{C} \backslash\{0\})$. 
To prove that $\varphi \in F M O(0)$, fix $N$ such that $(p-1) N>1$, and set $\varepsilon=\varepsilon_{N}=z_{N}+r_{N}$. Then $\bigcup_{n \geq N} D_{n} \subset \mathbb{D}(\varepsilon):=\{z \in \mathbb{C}:|z|<\varepsilon\}$ and

$$
\begin{aligned}
\int_{\mathbb{D}(\varepsilon)} \varphi=\sum_{n \geq N} \int_{D_{n}} \varphi=\pi \sum_{n \geq N} c_{n} r_{n}^{2}=\sum_{n \geq N} 2^{2(1-p) n^{2}} \\
<\sum_{n \geq N} 2^{2(1-p) n}<C \cdot\left[2^{(1-p) N}\right]^{2}<2 C \varepsilon^{2} .
\end{aligned}
$$

Hence $\varphi \in F M O(0)$ and, consequently, $\varphi \in F M O(\mathbb{C})$.

On the other hand

$$
\int_{\mathbb{D}(\varepsilon)} \varphi^{p}=\pi \sum_{n>N} c_{n}^{p} \cdot r_{n}^{2}=\sum_{n>N} 1=\infty .
$$

Hence $\varphi \notin L^{p}(\mathbb{D}(\varepsilon))$ and therefore $\varphi \notin B M O_{\text {loc }}$ because $\mathrm{BMO}_{\text {loc }} \subset L_{\text {loc }}^{p}$ for all $p \in[1, \infty)$.

Example 2.2. We conclude this section by constructing functions $\varphi$ : $\mathbb{C} \rightarrow \mathbb{R}$ of the class $C^{\infty}(\mathbb{C} \backslash\{0\})$ which belongs to FMO but not to $L_{\text {loc }}^{p}$ for any $p>1$ and hence not to $\mathrm{BMO}_{\text {loc }}$.

In this example, $p=1+\delta$ with an arbitrarily small $\delta>0$. Set

$$
\varphi_{0}(z)= \begin{cases}e^{\frac{1}{|z|^{2}-1}}, & \text { if }|z|<1 \\ 0, & \text { if }|z| \geq 1\end{cases}
$$

Then $\varphi_{0}$ belongs to $C_{0}^{\infty}$ and hence to $B M O_{\text {loc }}$. Consider the function

$$
\varphi(z)= \begin{cases}\varphi_{k}(z), & \text { if } z \in B_{k}, \\ 0, & \text { if } z \in \mathbb{C} \backslash \cup B_{k}\end{cases}
$$

where $B_{k}=B\left(z_{k}, r_{k}\right), z_{k}=2^{-k}, r_{k}=2^{-(1+\delta) k^{2}}, \delta>0$, and

$$
\varphi_{k}(z)=2^{2 k^{2}} \varphi_{0}\left(\frac{z-z_{k}}{r_{k}}\right), \quad z \in B_{k}, k=2,3, \ldots
$$

Then $\varphi$ is smooth in $\mathbb{C} \backslash\{0\}$ and, thus, belongs to $\mathrm{BMO}_{\text {loc }}(\mathbb{C} \backslash\{0\})$, and hence to $\operatorname{FMO}(\mathbb{C} \backslash\{0\})$.

Now,

$$
\int_{B_{k}} \varphi_{k}(z) d m(z)=2^{-2 \delta k^{2}} \int_{\mathbb{C}} \varphi_{0}(z) d m(z) .
$$

Setting

$$
K=K(\varepsilon)=\left[\log _{2} \frac{1}{\varepsilon}\right] \leq \log _{2} \frac{1}{\varepsilon}
$$


where $[A]$ denotes the integral part of the number $A$, we have

$$
J=f_{D(\varepsilon)} \varphi(z) d m(z) \leq I \cdot \sum_{k=K}^{\infty} 2^{-2 \delta k^{2}} / \pi 2^{-2(K+1)},
$$

where $I=\int_{\mathbb{C}} \varphi(z) d m(z)$. If $K \delta>1$, i.e. $K>1 / \delta$, then

$$
\sum_{k=K}^{\infty} 2^{-2 \delta k^{2}} \leq \sum_{k=K}^{\infty} 2^{-2 k}=2^{-2 K} \sum_{k=0}^{\infty}\left(\frac{1}{4}\right)^{k}=\frac{4}{3} \cdot 2^{-2 K},
$$

i.e., $J \leq 16 I / 3 \pi$. Hence

$$
\varlimsup_{\varepsilon \rightarrow 0} f_{B(\varepsilon)} \varphi(z) d m(z)<\infty .
$$

Thus, $\varphi \in$ FMO by Corollary 2.1.

On the other hand,

$$
\int_{B_{k}} \varphi_{k}^{1+\delta}(z) d m(z)=\int_{\mathbb{C}} \varphi_{0}^{1+\delta}(z) d m(z)
$$

and hence $\varphi \notin L^{1+\delta}(U)$ for any neighborhood $U$ of 0 .

\section{Convergence of the Sobolev functions}

Our approach for deriving criteria for existence of solutions to the Beltrami equations is approximative and based on the corresponding convergence theorems, the Arzela-Askoli theorem and the modulus techniques.

First of all, let us recall necessary definitions and basic facts concerning the Sobolev spaces $W^{l, p}$ and the classes $L^{p}, p \in[1, \infty]$. Given an open set $U$ in $\mathbb{R}^{n}$ and a natural number $l, C_{0}^{l}$ denotes a collection of all functions $\varphi: U \rightarrow \mathbb{R}$ with compact support having all partial continuous derivatives of order at most $l$ in $U . \varphi \in C_{0}^{\infty}$ if $\varphi \in C_{0}^{l}$ for all $l=1,2, \ldots$ A vector $\alpha=\left(\alpha_{1}, \ldots, \alpha_{n}\right)$ with natural coordinates is called a multiindex. Every multi-index $\alpha$ is associated with the differential operator $D^{\alpha}=\partial^{|\alpha|} / \partial x_{1}^{\alpha_{1}} \cdots \partial x_{n}^{\alpha_{n}}$ where $|\alpha|=\alpha_{1}+\cdots+\alpha_{n}$.

Now, let $u$ and $v: U \rightarrow \mathbb{R}$ be locally integrable functions. The function $v$ is called the distributional derivative $D^{\alpha} u$ of $u$ if

$$
\int_{\Omega} u D^{\alpha} \varphi d x=(-1)^{|\alpha|} \int_{\Omega} v \varphi d x \quad \forall \varphi \in C_{0}^{\infty}
$$


The concept of the distributional derivative was introduced by Sobolev in [95]. The Sobolev class $W^{l, p}(\Omega)$ consists of all functions $u: U \rightarrow \mathbb{R}$ in $L^{p}(U), p \geq 1$, with distributional derivatives of order $l$ summable of order $p$. A function $u: U \rightarrow \mathbb{R}$ belongs to $W_{\text {loc }}^{l, p}(U)$ if $u \in W^{l, p}\left(U_{*}\right)$ for every open set $U_{*}$ with compact closure $\overline{U_{*}} \subset U$. A similar notion introduced for vector-functions $f: U \rightarrow \mathbb{R}$ in the componentwise sense.

A function $\omega: \mathbb{R}^{n} \rightarrow \mathbb{R}$ with a compact support in $\mathbb{B}$ is called a Sobolev averaging kernel if $\omega$ is nonnegative, belongs to $C_{0}^{\infty}\left(\mathbb{R}^{n}\right)$ and

$$
\int_{\mathbb{R}^{n}} \omega(x) d x=1
$$

The well-know example of such a function is $\omega(x)=\gamma \varphi\left(|x|^{2}-\frac{1}{4}\right)$ where $\varphi(t)=e^{1 / t}$ for $t<0$ and $\varphi(t) \equiv 0$ for $t \geq 0$ and the constant $\gamma$ is chosen so that (3.2) holds. Later on, we use only $\omega$ depending on $|x|$.

Let $U$ be a nonempty bounded open subset of $\mathbb{R}$ and $f \in L^{1}(U)$. Extending $f$ by zero outside of $U$, we set

$$
f_{h}=\omega_{h} * f=\int_{|y| \leq 1} f(x+h y) \omega(y) d y=\frac{1}{h^{n}} \int_{U} f(z) \omega\left(\frac{z-x}{h}\right) d z
$$

where $f_{h}=\omega_{h} * f, \omega_{h}(y)=\omega(y / h), h>0$, is called the Sobolev mean function for $f$. It is known that $f_{h} \in C_{0}^{\infty}\left(\mathbb{R}^{n}\right),\left\|f_{h}\right\|_{p} \leq\|f\|_{p}$ for every $f \in L^{p}(U), p \in[1, \infty]$, and $f_{h} \rightarrow f$ in $L^{p}(U)$ for every $f \in L^{p}(U)$, $p \in[1, \infty)$, see e.g. 1.2.1 in [65]. It is clear that, if $f$ has a compact support in $U$, then $f_{h}$ also has a compact support in $U$ for small enough $h$.

A sequence $\varphi_{k} \in L^{1}(U)$ is called weakly fundamental if

$$
\lim _{k_{1}, k_{2} \rightarrow \infty} \int_{U} \Phi(x)\left(\varphi_{k_{1}}(x)-\varphi_{k_{2}}(x)\right) d x=0 \quad \forall \Phi \in L^{\infty}(U)
$$

It is well-known that the space $L^{1}(U)$ is weakly complete, i.e., every weakly fundamental sequence $\varphi_{k} \in L^{1}(U)$ converges weakly in $L^{1}(U)$, see e.g. Theorem IV.8.6 in [28].

It is known the following fact for the Sobolev classes $W^{1, p}(U), p>1$, see e.g. Lemma III.3.5 in [75], see also Theorem 4.6.1 in [31]. Note that this fact under $p=2$ was known long ago in the plane for the socalled mappings with the bounded Dirichlet integral, see e.g. Theorem 1 in [100]. 
Lemma 3.1. Let $U$ be a bounded open set in $\mathbb{R}^{n}$ and let $f_{k}: U \rightarrow \mathbb{R}$ be a sequence of functions of the class $W^{1, p}(U), p>1$. Suppose that the norm sequence $\left\|f_{k}\right\|_{1, p}$ is bounded and $f_{k} \rightarrow f$ as $k \rightarrow \infty$ in $L^{1}(U)$. Then $f \in W^{1, p}(U)$ and $\partial f_{k} / \partial x_{j} \rightarrow \partial f / \partial x_{j}$ as $k \rightarrow \infty$ weakly in $L^{p}(U)$.

In comparison with [78], we applied in [15,84,85] the following lemma instead of Lemma 3.1 which is not valid for $p=1$. Its proof is based just on the weak completeness of the space $L^{1}$, see [84] and [85].

Lemma 3.2. Let $U$ be a bounded open set in $\mathbb{R}$ and let $f_{k}: U \rightarrow \mathbb{R}$ be a sequence of functions of the class $W^{1,1}(U)$. Suppose that $f_{k} \rightarrow f$ as $k \rightarrow \infty$ weakly in $L^{1}(U), \partial f_{k} / \partial x_{j}, k=1,2, \ldots, j=1,2, \ldots, n$ are uniformly bounded in $L^{1}(U)$ and their indefinite integrals are absolutely equicontinuous. Then $f \in W^{1,1}(U)$ and $\partial f_{k} / \partial x_{j} \rightarrow \partial f / \partial x_{j}$ as $k \rightarrow \infty$ weakly in $L^{1}(U)$.

Remark 3.1. The weak convergence $f_{k} \rightarrow f$ in $L^{1}(U)$ implies that

$$
\sup _{k}\left\|f_{k}\right\|_{1}<\infty
$$

see e.g. IV.8.7 in [28]. The latter together with

$$
\sup _{k}\left\|\partial f_{k} / \partial x_{j}\right\|_{1}<\infty
$$

$j=1,2, \ldots, n$, implies that $f_{k} \rightarrow f$ by the norm in $L^{q}$ for every $1<q<n /(n-1)$, the limit function $f$ belongs to $B V(U)$, the class of functions of bounded variation, but, generally speaking, not to the class $W^{1,1}(U)$, see e.g. Remark in 4.6 and Theorem 5.2.1 in [31]. Thus, the additional condition of Lemma 3.2 on absolute equicontinuity of the indefinite integrals of $\partial f_{k} / \partial x_{j}$ is essential, cf. also Remark to Theorem I.2.4 in [75].

The next convergence theorem for ACL homeomorphisms was first proved on the base of Lemma 3.2 in [84], cf. also [85].

Theorem 3.1. Let $D$ be a domain in $\mathbb{C}$ and let $f_{n}: D \rightarrow \mathbb{C}$ be a sequence of ACL homeomorphisms with complex dilatations $\mu_{n}$ such that

$$
\frac{1+\left|\mu_{n}(z)\right|}{1-\left|\mu_{n}(z)\right|} \leq Q(z) \in L_{\text {loc }}^{1} \quad \forall n=1,2, \ldots
$$

If $f_{n} \rightarrow f$ uniformly on each compact set in $D$, where $f$ is a homeomorphism, then $f \in \mathrm{ACL}$ and $\partial f_{n}$ and $\bar{\partial} f_{n}$ converge weakly in $L_{\mathrm{loc}}^{1}$ to $\partial f$ and $\bar{\partial} f$, respectively. Moreover, if in addition $\mu_{n} \rightarrow \mu$ a.e., then $\bar{\partial} f_{n}=\mu \partial f_{n}$ a.e. 
Remark 3.2. In fact, it is easy to show that under the condition (3.4) $f_{n}$ as well as $f$ belong to $W_{\text {loc }}^{1,1}$. Moreover, if in addition $Q \in L_{\text {loc }}^{p}, p>1$, then $f_{n}$ and $f$ belong to $W_{\text {loc }}^{1, s}, \partial f_{n} \rightarrow \partial f$ and $\bar{\partial} f_{n} \rightarrow \bar{\partial} f$ weakly in $L_{\text {loc }}^{s}$ where $s=2 p /(1+p)$, see Lemma 3.1.

Proposition 3.1. Let $D$ be a domain in $\overline{\mathbb{C}}$ and $f_{n}: D \rightarrow \overline{\mathbb{C}}, n=1,2, \ldots$, a sequence of homeomorphisms such that $f_{n} \rightarrow f$ uniformly on compact sets in $D$ with respect to the spherical (chordal) metric. If the limit function $f$ is discrete, then $f$ is a homeomorphism.

Corollary 3.1. Let $D$ be a domain in $\mathbb{C}$ and $f_{n}: D \rightarrow \overline{\mathbb{C}}, n=1,2, \ldots$, a sequence of quasiconformal mappings which satisfy (3.4). If $f_{n} \rightarrow f$ locally uniformly, then either $f$ is constant or $f$ is an ACL homeomorphism and $\partial f_{n}$ and $\bar{\partial} f_{n}$ converge weakly in $L_{\mathrm{loc}}^{1}\left(D \backslash\left\{f^{-1}(\infty)\right\}\right)$ to $\partial f$ and $\bar{\partial} f$, respectively. If in addition, $\mu_{n} \rightarrow \mu$ a.e., then $\bar{\partial} f=\mu \partial f$ a.e.

The following theorem in [15] is a generalization of the Bojarski convergence result in [10], Lemma 4.2, where $Q$ was in $L^{\infty}$.

Theorem 3.2. Let $D$ be a domain in $\mathbb{C}$ and let $f_{n}: D \rightarrow \mathbb{C}$ be a sequence of homeomorphic $W_{\mathrm{loc}}^{1,1}(D)$ solutions of the equations $\bar{\partial} f_{n}=$ $\mu_{n} \partial f_{n}+\nu_{n} \overline{\partial f_{n}}$ with $\left|\mu_{n}(z)\right|+\left|\nu_{n}(z)\right|<1$ a.e. such that

$$
\frac{1+\left|\mu_{n}(z)\right|+\left|\nu_{n}(z)\right|}{1-\left|\mu_{n}(z)\right|-\left|\nu_{n}(z)\right|} \leq Q(z) \in L_{\mathrm{loc}}^{1}(D) \quad \forall n=1,2, \ldots
$$

If $f_{n} \rightarrow f$ uniformly on each compact set in $D$ where $f: D \rightarrow \mathbb{C}$ is a homeomorphism, then $f \in W_{\mathrm{loc}}^{1,1}(D)$ and $\partial f_{n} \rightarrow \partial f$ and $\bar{\partial} f_{n} \rightarrow \bar{\partial} f$ weakly in $L_{\mathrm{loc}}^{1}(D)$. Moreover, if in addition $\mu_{n} \rightarrow \mu$ and $\nu_{n} \rightarrow \nu$ a.e., then $\bar{\partial} f=\mu \partial f+\nu \overline{\partial f}$ a.e.

The kernel of a sequence of open sets $\Omega_{n} \subseteq \overline{\mathbb{C}}, n=1,2, \ldots$ is the open set

$$
\Omega_{0}=\operatorname{Kern} \Omega_{n}:=\bigcup_{m=1}^{\infty} \operatorname{Int}\left(\bigcap_{n=m}^{\infty} \Omega_{n}\right)
$$

where $\operatorname{Int} A$ denotes the set consisting of all inner points of $A$, in other words, Int $A$ is the union of all open disks in $A$ with respect to the spherical distance.

Proposition 3.2. Let $h_{n}: D \rightarrow D_{n}^{\prime}, D_{n}^{\prime}=h_{n}(D)$, be a sequence of homeomorphisms given in a domain $D \subseteq \overline{\mathbb{C}}$. If $h_{n}$ converge as $n \rightarrow$ $\infty$ locally uniformly with respect to the spherical (chordal) metric to a homeomorphism $h: D \rightarrow D^{\prime} \subseteq \overline{\mathbb{C}}$, then $D^{\prime}=h(D) \subseteq \operatorname{Kern} D_{n}^{\prime}$. 
Remark 3.3. In particular, Proposition 3.2 implies that $h(D) \subseteq \mathbb{C}$ if $h_{n}(D) \subseteq \mathbb{C}$ for all $n=1,2, \ldots$

Lemma 3.3. Let $D$ be a domain in $\overline{\mathbb{C}}$ and let $f_{n}: D \rightarrow \overline{\mathbb{C}}$ be a sequence of homeomorphisms from $D$ into $\overline{\mathbb{C}}$ such that $f_{n} \rightarrow f$ as $n \rightarrow \infty$ locally uniformly with respect to the spherical metric to a homeomorphism $f$ from $D$ into $\overline{\mathbb{C}}$. Then $f_{n}^{-1} \rightarrow f^{-1}$ locally uniformly in $f(D)$, too.

Corollary 3.2. Let $D$ be a domain in $\mathbb{C}$ and $f_{n}: D \rightarrow \mathbb{C}, n=1,2, \ldots$, a sequence of quasiconformal mappings which satisfies (3.5). If $f_{n} \rightarrow f$ locally uniformly where $f$ is a homeomorphism, then $f^{-1} \in W_{\mathrm{loc}}^{1,2}$.

Corollary 3.3. Let $D$ be a domain in $\mathbb{C}$ and $f_{n}: D \rightarrow \mathbb{C}, n=1,2, \ldots$, be a sequence of quasiconformal mappings which satisfy the equations $\bar{\partial} f_{n}=\mu_{n} \partial f_{n}+\nu_{n} \overline{\partial f_{n}}$ with $\left|\mu_{n}(z)\right|+\left|\nu_{n}(z)\right|<1$ a.e. and the condition (3.5). If $f_{n} \rightarrow f$ locally uniformly with respect to the spherical (chordal) metric, then either $f$ is constant or $f$ is a homeomorphism of the class $W_{\mathrm{loc}}^{1,1}(D)$ and $\partial f_{n} \rightarrow \partial f$ and $\bar{\partial} f_{n} \rightarrow \bar{\partial} f$ weakly in $L_{\mathrm{loc}}^{1}(D)$. Moreover, if in addition $\mu_{n} \rightarrow \mu$ and $\nu_{n} \rightarrow \nu$ a.e., then $\bar{\partial} f=\mu \partial f+\nu \overline{\partial f}$ a.e.

\section{Equivalent integral conditions}

Many existence theorems for the Beltrami equation are given in terms of integral restrictions on the dilatations $K_{\mu}(z)$ and $K_{\mu}^{T}\left(z, z_{0}\right)$. In [84] and [85], we have established equivalence of some integral conditions.

For every non-decreasing function $\Phi:[0, \infty] \rightarrow[0, \infty]$, the inverse $\Phi^{-1}:[0, \infty] \rightarrow[0, \infty]$ can be well defined by setting

$$
\Phi^{-1}(\tau)=\inf _{\Phi(t) \geq \tau} t
$$

Here inf is equal to $\infty$ if the set of $t \in[0, \infty]$ such that $\Phi(t) \geq \tau$ is empty. Note that the function $\Phi^{-1}$ is non-decreasing, too.

Remark 4.1. It is evident immediately by the definition that

$$
\Phi^{-1}(\Phi(t)) \leq t \quad \forall t \in[0, \infty]
$$

with the equality in (4.2) except intervals of constancy of the function $\Phi(t)$.

Further, the integral in (4.5) is understood as the Lebesgue-Stieltjes integral and the integrals in (4.4) and (4.6)-(4.9) as the ordinary Lebesgue integrals. In (4.4) and (4.5) we complete the definition of integrals by $\infty$ if $\Phi(t)=\infty$, correspondingly, $H(t)=\infty$, for all $t \geq$ $T \in[0, \infty)$. 
Theorem 4.1. Let $\Phi:[0, \infty] \rightarrow[0, \infty]$ be a non-decreasing function and set

$$
H(t)=\log \Phi(t) .
$$

Then the equality

$$
\int_{\Delta}^{\infty} H^{\prime}(t) \frac{d t}{t}=\infty
$$

implies the equality

$$
\int_{\Delta}^{\infty} \frac{d H(t)}{t}=\infty
$$

and (4.5) is equivalent to every of the equalities:

$$
\int_{\Delta}^{\infty} H(t) \frac{d t}{t^{2}}=\infty
$$

for some $\Delta>0$,

$$
\int_{0}^{\delta} H\left(\frac{1}{t}\right) d t=\infty
$$

for some $\delta>0$,

$$
\int_{\Delta_{*}}^{\infty} \frac{d \eta}{H^{-1}(\eta)}=\infty
$$

for some $\Delta_{*}>H(+0)$,

$$
\int_{\delta_{*}}^{\infty} \frac{d \tau}{\tau \Phi^{-1}(\tau)}=\infty
$$

for some $\delta_{*}>\Phi(+0)$.

Moreover, (4.4) is equivalent to (4.5) and hence (4.4)-(4.9) are equivalent each to other if $\Phi$ is in addition absolutely continuous. In particular, all the conditions (4.4)-(4.9) are equivalent if $\Phi$ is convex and non-decreasing.

Remark 4.2. We have in mind $+\infty$ at the right hand sides of the conditions (4.4)-(4.9). If $\Phi(t)=0$ for $t \in\left[0, t_{*}\right]$, then $H(t)=-\infty$ for $t \in\left[0, t_{*}\right]$ and we complete the definition $H^{\prime}(t)=0$ for $t \in\left[0, t_{*}\right]$. Note, the conditions (4.5) and (4.6) exclude that $t_{*}$ belongs to the interval of integrability because in the contrary case the left hand sides in (4.5) and 
(4.6) are either equal to $-\infty$ or indeterminate. Hence we may assume in (4.4)-(4.7) that $\Delta>t_{0}$ where $t_{0}:=\sup _{\Phi(t)=0} t, t_{0}=0$ if $\Phi(0)>0$, and $\delta<1 / t_{0}$, correspondingly.

Recall that a function $\psi:[0, \infty] \rightarrow[0, \infty]$ is called convex if $\psi\left(\lambda t_{1}+\right.$ $\left.(1-\lambda) t_{2}\right) \leq \lambda \psi\left(t_{1}\right)+(1-\lambda) \psi\left(t_{2}\right)$ for all $t_{1}$ and $t_{2} \in[0, \infty]$ and $\lambda \in[0,1]$.

In what follows, $\mathbb{D}$ denotes the unit disk in the complex plane $\mathbb{C}$,

$$
\mathbb{D}=\{z \in \mathbb{C}:|z|<1\} .
$$

Lemma 4.1. Let $Q: \mathbb{D} \rightarrow[0, \infty]$ be a measurable function and let $\Phi:$ $[0, \infty] \rightarrow[0, \infty]$ be a non-decreasing convex function. Then

$$
\int_{0}^{1} \frac{d r}{r q(r)} \geq \frac{1}{2} \int_{N}^{\infty} \frac{d \tau}{\tau \Phi^{-1}(\tau)}
$$

where $q(r)$ is the average of the function $Q(z)$ over the circle $|z|=r$ and

$$
N=\int_{\mathbb{D}} \Phi(Q(z)) d m(z) .
$$

Theorem 4.2. Let $Q: \mathbb{D} \rightarrow[0, \infty]$ be a measurable function such that

$$
\int_{\mathbb{D}} \Phi(Q(z)) d m(z)<\infty
$$

where $\Phi:[0, \infty] \rightarrow[0, \infty]$ is a non-decreasing convex function such that

$$
\int_{\delta_{0}}^{\infty} \frac{d \tau}{\tau \Phi^{-1}(\tau)}=\infty
$$

for some $\delta_{0}>\tau_{0}:=\Phi(0)$. Then

$$
\int_{0}^{1} \frac{d r}{r q(r)}=\infty
$$

where $q(r)$ is the average of the function $Q(z)$ over the circle $|z|=r$.

Remark 4.3. Note that (4.14) implies that

$$
\int_{\delta}^{\infty} \frac{d \tau}{\tau \Phi^{-1}(\tau)}=\infty
$$


for every $\delta \in[0, \infty)$ but (4.16) for some $\delta \in[0, \infty)$, generally speaking, does not imply (4.14). Indeed, for $\delta \in\left[0, \delta_{0}\right)$, (4.14) evidently implies (4.16) and, for $\delta \in\left(\delta_{0}, \infty\right)$, we have that

$$
0<\int_{\delta_{0}}^{\delta} \frac{d \tau}{\tau \Phi^{-1}(\tau)} \leq \frac{1}{\Phi^{-1}\left(\delta_{0}\right)} \log \frac{\delta}{\delta_{0}}<\infty
$$

because $\Phi^{-1}$ is non-decreasing and $\Phi^{-1}\left(\delta_{0}\right)>0$. Moreover, by the definition of the inverse function $\Phi^{-1}(\tau) \equiv 0$ for all $\tau \in\left[0, \tau_{0}\right], \tau_{0}=\Phi(0)$, and hence (4.16) for $\delta \in\left[0, \tau_{0}\right)$, generally speaking, does not imply (4.14). If $\tau_{0}>0$, then

$$
\int_{\delta}^{\tau_{0}} \frac{d \tau}{\tau \Phi^{-1}(\tau)}=\infty \quad \forall \delta \in\left[0, \tau_{0}\right)
$$

However, (4.18) gives no information on the function $Q(z)$ itself and, consequently, (4.16) for $\delta<\Phi(0)$ cannot imply (4.15) at all.

By (4.16) the proof of Theorem 4.2 is reduced to Lemma 4.1. Combining Theorems 4.1 and 4.2 we also obtain the following conclusion.

Corollary 4.1. If $\Phi:[0, \infty] \rightarrow[0, \infty]$ is a non-decreasing convex function and $Q$ satisfies the condition (4.13), then every of the conditions (4.4)-(4.9) implies (4.15).

\section{General Beltrami equation}

Following [15], we call a homeomorphism $f \in W_{\text {loc }}^{1,1}(D)$ a regular solution of (1.4) if $f$ satisfies (1.4) and the Jacobian $J_{f}(z) \neq 0$ almost everywhere in $D$. This and next sections contain new criteria for existence of just regular solutions to the general Beltrami equations (1.4), see [15] and [16].

Theorem 5.1. Let $D$ be a domain in $\mathbb{C}$ and let $\mu$ and $\nu: D \rightarrow \mathbb{C}$ be measurable functions with $|\mu(z)|+|\nu(z)|<1$ a.e. such that

$$
K_{\mu, \nu}(z)=\frac{1+|\mu(z)|+|\nu(z)|}{1-|\mu(z)|-|\nu(z)|} \leq Q(z) \in F M O(D) .
$$

Then the Beltrami equation (1.4) has a regular solution.

Note that, y a result of Hencl and Koskela [42], it follows that the inverse mapping $f^{-1} \in W_{\text {loc }}^{1,2}$ for a regular solution $f$.

Theorem 5.1 implies the existence theorem for the case of majorants $Q(z)$ in the class BMO, see [14]. Moreover, in this case $f \in W_{\text {loc }}^{1, s}$ for all $1 \leq s<2$. 
Corollary 5.1. If

$$
\varlimsup_{\varepsilon \rightarrow 0} \underset{B\left(z_{0}, \varepsilon\right)}{f} \frac{1+|\mu(z)|+|\nu(z)|}{1-|\mu(z)|-|\nu(z)|} d m(z)<\infty \quad \forall z_{0} \in D
$$

then (1.4) has a regular solution.

The following theorem is a source for deriving various integral criteria for the existence of regular solutions to (1.4).

Theorem 5.2. Let $D$ be a domain in $\mathbb{C}$ and let $\mu$ and $\nu: D \rightarrow \mathbb{C}$ be measurable functions with $|\mu(z)|+|\nu(z)|<1$ a.e. and $K_{\mu, \nu} \in L_{\mathrm{loc}}^{1}(D)$. Suppose that

$$
\int_{0}^{\delta\left(z_{0}\right)} \frac{d r}{r k_{z_{0}}(r)}=\infty \quad \forall z_{0} \in D
$$

where $\delta\left(z_{0}\right)<\operatorname{dist}\left(z_{0}, \partial D\right)$ and $k_{z_{0}}(r)$ is the mean of $K_{\mu, \nu}(z)$ over $\left|z-z_{0}\right|=r$. Then the Beltrami equation (1.4) has a regular solution.

Corollary 5.2. In particular, if

$$
k_{z_{0}}(r)=O\left(\log \frac{1}{r}\right) \quad \text { as } r \rightarrow 0 \quad \forall z_{0} \in D
$$

then (1.4) has a regular solution.

In fact, the condition (5.3) implies the whole scale of conditions in terms of the iterated logarithms, see [38].

Remark 5.1. The case $\infty \in D$ is included in our scheme by the standard way. The enumerated conditions for $K_{\mu, \nu}(z)$ at $\infty$ should be understood as the corresponding conditions for $K_{\mu, \nu}(1 / \bar{z})$ at 0 . Of course, all these latter conditions can also be written explicitly in terms of $K_{\mu, \nu}(z)$ itself after the change of variables $z \longmapsto 1 / \bar{z}$.

For example, the concept of finite mean oscillation can also be extended to infinity in the following way. Given a domain $D$ in the extended complex plane $\overline{\mathbb{C}}, \infty \in D$, and a function $\varphi: D \rightarrow \mathbb{R}$, we say that $\varphi$ has finite mean oscillation at $\infty$ if the function $\varphi^{*}(z)=\varphi(1 / \bar{z})$ has finite mean oscillation at 0 . Clearly, by the change of variables $z \longmapsto 1 / \bar{z}$ the latter is equivalent to the condition

$$
\int_{|z| \geq R}\left|\varphi(z)-\tilde{\varphi}_{R}\right| \frac{d m(z)}{|z|^{4}}=O\left(\frac{1}{R^{2}}\right)
$$


where

$$
\tilde{\varphi}_{R}=\frac{R^{2}}{\pi} \int_{|z| \geq R} \varphi(z) \frac{d m(z)}{|z|^{4}} .
$$

The condition (5.3) at $\infty$ has the form

$$
\int_{\Delta}^{\infty} \frac{d R}{R K(R)}=\infty
$$

for some $\Delta>0$ where $K(R)$ is the average of $K_{\mu, \nu}(z)$ over the circle $|z|=R$. The condition (5.4) at $\infty$ is written as

$$
K(R)=O(\log R) \quad \text { as } R \rightarrow \infty .
$$

Theorem 5.2 and Corollary 4.1 yield the next result.

Theorem 5.3. Let $D$ be a domain in $\mathbb{C}$ and let $\mu$ and $\nu: D \rightarrow \mathbb{C}$ be measurable functions with $|\mu(z)|+|\nu(z)|<1$ a.e. such that

$$
\int_{D} \Phi\left(K_{\mu, \nu}(z)\right) d m(z)<\infty
$$

where $\Phi:[0, \infty] \rightarrow[0, \infty]$ is a non-decreasing convex function. If $\Phi$ satisfies at least one of the conditions (4.4)-(4.9), then the Beltrami equation (1.4) has a regular solution.

Remark 5.2. The condition (5.9) can be also localized to neighborhoods $U_{z_{0}}$ of points $z_{0} \in D$ with $\Phi=\Phi_{z_{0}}$ under the same conditions on the functions $\Phi_{z_{0}}$. If $\infty \in D$, then the condition (5.9) for $K_{\mu, \nu}(z)$ at $\infty \in D$ should be understood as the corresponding condition for $K_{\mu, \nu}(1 / \bar{z})$ at 0 . The latter condition can also be written explicitly in terms of $K_{\mu, \nu}(z)$ itself after the change of variables $z \longmapsto 1 / \bar{z}$ in the form

$$
\int_{U_{\infty}} \Phi_{\infty}\left(K_{\mu, \nu}(z)\right) \frac{d m(z)}{|z|^{4}}<\infty .
$$

If the domain $D$ is unbounded, then it is better to use the global condition

$$
\int_{D} \Phi\left(K_{\mu, \nu}(z)\right) \frac{d m(z)}{\left(1+|z|^{2}\right)^{2}}<\infty
$$

instead of (5.9).

We may assume in the above theorem that the functions $\Phi_{z_{0}}(t)$ and $\Phi(t)$ are not convex and non-decreasing on the whole segment $[0, \infty]$ but 
only on a segment $[T, \infty]$ for some $T \in(1, \infty)$. Indeed, every function $\Phi:[0, \infty] \rightarrow[0, \infty]$ which is convex and non-decreasing on a segment $[T, \infty], T \in(0, \infty)$, can be replaced by a non-decreasing convex function $\Phi_{T}:[0, \infty] \rightarrow[0, \infty]$ in the following way. We set $\Phi_{T}(t) \equiv 0$ for all $t \in[0, T], \Phi(t)=\varphi(t), t \in\left[T, T_{*}\right]$, and $\Phi_{T} \equiv \Phi(t), t \in\left[T_{*}, \infty\right]$, where $\tau=\varphi(t)$ is the line passing through the point $(0, T)$ and touching upon the graph of the function $\tau=\Phi(t)$ at a point $\left(T_{*}, \Phi\left(T_{*}\right)\right), T_{*} \geq T$. For such a function we have by the construction that $\Phi_{T}(t) \leq \Phi(t)$ for all $t \in[1, \infty]$ and $\Phi_{T}(t)=\Phi(t)$ for all $t \geq T_{*}$.

\section{Consequences for reduced Beltrami equations}

The equation of the form

$$
f_{\bar{z}}=\lambda(z) \operatorname{Re} f_{z}
$$

with $|\lambda(z)|<1$ a.e. is called the reduced Beltrami equation, see e.g. $[5,10-13,109]$. The equation (6.1) can be rewritten as the equation (1.4) with

$$
\mu(z)=\nu(z)=\frac{\lambda(z)}{2}
$$

and then

$$
K_{\mu, \nu}(z)=K_{\lambda}(z):=\frac{1+|\lambda(z)|}{1-|\lambda(z)|} .
$$

Thus, the results of the previous sections give the following consequences for the reduced Beltrami equations (6.1).

Theorem 6.1. Let $D$ be a domain in $\mathbb{C}$ and let $\lambda: D \rightarrow \mathbb{C}$ be a measurable function with $|\lambda(z)|<1$ a.e. such that

$$
K_{\lambda}(z)=\frac{1+|\lambda(z)|}{1-|\lambda(z)|} \leq Q(z) \in F M O(D) .
$$

Then the Beltrami equation (6.1) has a regular solution.

Corollary 6.1. In particular, if

$$
\varlimsup_{\varepsilon \rightarrow 0} \int_{B\left(z_{0}, \varepsilon\right)} \frac{1+|\lambda(z)|}{1-|\lambda(z)|} d m(z)<\infty \quad \forall z_{0} \in D
$$

then (6.1) has a regular solution. 
Theorem 6.2. Let $D$ be a domain in $\mathbb{C}$ and let $\lambda: D \rightarrow \mathbb{C}$ be a measurable function with $|\lambda(z)|<1$ a.e. and $K_{\lambda} \in L_{\mathrm{loc}}^{1}(D)$. Suppose that

$$
\int_{0}^{\delta\left(z_{0}\right)} \frac{d r}{r \Lambda_{z_{0}}(r)}=\infty \quad \forall z_{0} \in D
$$

where $\delta\left(z_{0}\right)<\operatorname{dist}\left(z_{0}, \partial D\right)$ and $\Lambda_{z_{0}}(r)$ is the mean of $K_{\lambda}(z)$ over $\left|z-z_{0}\right|=$ $r$. Then the Beltrami equation (6.1) has a regular solution.

Corollary 6.2. In particular, if

$$
\Lambda_{z_{0}}(r)=O\left(\log \frac{1}{r}\right) \quad \text { as } r \rightarrow 0 \quad \forall z_{0} \in D
$$

then (6.1) has a regular solution.

From Theorem 5.3 we obtain the following consequence for the reduced Beltrami equations (6.1).

Theorem 6.3. Let $D$ be a domain in $\mathbb{C}$ and let $\lambda$ be a measurable function with $|\lambda(z)|<1$ a.e. such that

$$
\int_{D} \Phi\left(K_{\lambda}(z)\right) d m(z)<\infty
$$

where $\Phi:[0, \infty] \rightarrow[0, \infty]$ is a non-decreasing convex function. If $\Phi$ satisfies at least one of the conditions (4.4)-(4.9), then the reduced Beltrami equation (6.1) has a regular solution.

Remark 6.1. Remarks 5.2 are valid for the reduced Beltrami equation. Moreover, the above results remain true for the case in (1.4) when

$$
\nu(z)=\mu(z) e^{i \theta(z)}
$$

with an arbitrary measurable function $\theta(z): D \rightarrow \mathbb{R}$ and, in particular, for the equations of the form

$$
f_{\bar{z}}=\lambda(z) \operatorname{Im} f_{z}
$$

with a measurable coefficient $\lambda: D \rightarrow \mathbb{C},|\lambda(z)|<1$ a.e.

Next, note that we have constructed the examples showing that the conditions (4.4)-(4.9) are not only sufficient but also necessary for the Beltrami equations with two characteristics (1.4) and, in particular, for the reduced Beltrami equations (6.1) to have regular solutions. 


\section{On ring $Q$-homeomorphisms}

It is well-known that every quasiconformal mapping of a disk onto itself can be extended to a homeomorphism of the closed disk. The situation is different if we study more general homeomorphisms. Indeed, the homeomorphism $f: \mathbb{D} \rightarrow \mathbb{D}$ determined by

$$
f\left(r e^{i \theta}\right)=z e^{i \log (1-|z|)}
$$

has no limit as $|z| \rightarrow 1$. In Section 12 we will study the Dirichlet problem for the degenerate Beltrami equation. There we find sufficient conditions in terms of the Beltrami coefficient which guarantee the existence of regular solutions to the degenerate Beltrami equation with a homeomorphic extension to the boundary. Below we consider the so-called ring $Q$-homeomorphisms whose boundary behavior have been studied in [59].

Recall that, given a family of paths $\Gamma$ in $\overline{\mathbb{C}}$, a Borel function $\rho: \overline{\mathbb{C}} \rightarrow$ $[0, \infty]$ is called admissible for $\Gamma$, abbr. $\rho \in \operatorname{adm} \Gamma$, if

$$
\int_{\gamma} \rho(z)|d z| \geq 1
$$

for each $\gamma \in \Gamma$. The modulus of $\Gamma$ is defined by

$$
M(\Gamma)=\inf _{\rho \in \operatorname{adm} \Gamma} \int_{\mathbb{C}} \rho^{2}(z) d m(z) .
$$

Given a domain $D$ and two sets $E$ and $F$ in $\overline{\mathbb{C}}, \Gamma(E, F, D)$ denotes the family of all paths $\gamma:[a, b] \rightarrow \overline{\mathbb{C}}$ which join $E$ and $F$ in $D$, i.e., $\gamma(a) \in$ $E, \gamma(b) \in F$ and $\gamma(t) \in D$ for $a<t<b$. We set $\Gamma(E, F)=\Gamma(E, F, \overline{\mathbb{C}})$ if $D=\overline{\mathbb{C}}$. A ring domain, or shortly a ring in $\overline{\mathbb{C}}$ is a domain $R$ in $\overline{\mathbb{C}}$ whose complement consists of two components. Let $R$ be a ring in $\overline{\mathbb{C}}$. If $C_{1}$ and $C_{2}$ are the components of $\overline{\mathbb{C}} \backslash R$, we write $R=R\left(C_{1}, C_{2}\right)$. It is known that $M\left(\Gamma\left(C_{1}, C_{2}, R\right)\right)=\operatorname{cap} R\left(C_{1}, C_{2}\right)$, see e.g. [32]. Note also that $M\left(\Gamma\left(C_{1}, C_{2}, R\right)\right)=M\left(\Gamma\left(C_{1}, C_{2}\right)\right)$, see e.g. Theorem 11.3 in [106]. In what follows, we use the notations $B\left(z_{0}, r\right)$ and $C\left(z_{0}, r\right)$ for the open disk and the circle, respectively, in $\mathbb{C}$ centered at $z_{0} \in \mathbb{C}$ with the radius $r>0$ and $A\left(z_{0}, r_{1}, r_{2}\right)$ for the ring $\left\{z \in \mathbb{C}: r_{1}<\left|z-z_{0}\right|<r_{2}\right\}$.

Motivated by the ring definition of quasiconformality in [33], we introduced in [81], cf. also [80], the following notion that localizes and extends the notion of a $Q$-homeomorphism, see e.g. [63].

Let $D$ be a domain in $\mathbb{C}, z_{0} \in D, r_{0} \leq \operatorname{dist}\left(z_{0}, \partial D\right)$ and $Q$ : $B\left(z_{0}, r_{0}\right) \rightarrow[0, \infty]$ a measurable function. A homeomorphism $f: D \rightarrow \overline{\mathbb{C}}$ 
is called a ring $Q$-homeomorphism at the point $z_{0} \in D$ if

$$
M\left(\Gamma\left(f C_{1}, f C_{2}\right)\right) \leq \int_{A} Q(z) \cdot \eta^{2}\left(\left|z-z_{0}\right|\right) d m(z)
$$

for every ring $A=A\left(z_{0}, r_{1}, r_{2}\right), 0<r_{1}<r_{2}<r_{0}, C_{i}=C\left(z_{0}, r_{i}\right), i=1,2$, and for every measurable function $\eta:\left(r_{1}, r_{2}\right) \rightarrow[0, \infty]$ such that

$$
\int_{r_{1}}^{r_{2}} \eta(r) d r=1
$$

This notion was first extended to the boundary points in [85]. More precisely, given a domain $D$ in $\mathbb{C}$ and a measurable function $Q: D \rightarrow$ $[0, \infty]$, we say that a homeomorphism $f: D \rightarrow \overline{\mathbb{C}}$ is a ring $Q$-homeomorphism at a boundary point $z_{0}$ of the domain $D$ if

$$
M\left(\Delta\left(f C_{1}, f C_{2}, f D\right)\right) \leq \int_{A \cap D} Q(z) \cdot \eta^{2}\left(\left|z-z_{0}\right|\right) d m(z)
$$

for every ring $A=A\left(z_{0}, r_{1}, r_{2}\right)$ and every continua $C_{1}$ and $C_{2}$ in $D$ which belong to the different components of the complement to the ring $A$ in $\overline{\mathbb{C}}$, containing $z_{0}$ and $\infty$, correspondingly, and for every measurable function $\eta:\left(r_{1}, r_{2}\right) \rightarrow[0, \infty]$ satisfying the condition (7.4).

The following convergence theorem was first proved in [84], see also [85].

Theorem 7.1. Let $f_{n}: D \rightarrow \overline{\mathbb{C}}, n=1,2, \ldots$, be a sequence of ring $Q$ homeomorphisms at a point $z_{0} \in \bar{D}$. If $f_{n}$ converge locally uniformly to a homeomorphism $f: D \rightarrow \overline{\mathbb{C}}$, then $f$ is also a ring $Q$-homeomorphism at $z_{0}$.

\section{On strong ring solutions}

In this section we study the strong ring solutions which allow us to study some boundary problems. In [85-87], we have proved a series of criteria for the existence of strong ring solutions, in particular, in terms of finite mean oscillation majorants for tangential dilatations. Moreover, we have derived an extension of the well-known Lehto existence theorem. It is shown that the latter implies many known and new results on the existence of ACL homeomorphic solutions to the degenerate Beltrami equations, see Sections 8-10 and comments in Section 13. 
Below we will use the tangential dilatation with respect to a point $z_{0} \in \bar{D}$ which is defined by

$$
K_{\mu}^{T}\left(z, z_{0}\right)=\frac{\left|1-\frac{\overline{z-z_{0}}}{z-z_{0}} \mu(z)\right|^{2}}{1-|\mu(z)|^{2}},
$$

cf. $[4,37,38,57,80,81,88]$. Note that

$$
\frac{1}{K_{\mu}(z)} \leq K_{\mu}^{T}\left(z, z_{0}\right) \leq K_{\mu}(z) \quad \text { a.e. }
$$

Thus, $K_{\mu}^{T}\left(z, z_{0}\right) \neq 0$ and $\infty$ a.e. if $K_{\mu}(z)$ is locally integrable.

A homeomorphism $f: D \rightarrow \mathbb{C}$ is called a strong ring solution of the Beltrami equation (1.1) if $f$ is a regular solution and, in addition, $f$ is a ring $Q$-homeomorphism at every point $z_{0} \in \bar{D}$ with $Q(z)=K_{\mu}^{T}\left(z, z_{0}\right)$.

We show that the strong ring solutions exist for wide classes of the degenerate Beltrami equations.

Below we will use the tangential dilatation with respect to a point $z_{0} \in \bar{D}$ which is defined by

$$
K_{\mu}^{T}\left(z, z_{0}\right)=\frac{\left|1-\overline{z-z_{0}} \mu(z)\right|^{2}}{1-|\mu(z)|^{2}},
$$

cf. $[4,37,38,57,80,81,88]$. Note that

$$
\frac{1}{K_{\mu}(z)} \leq K_{\mu}^{T}\left(z, z_{0}\right) \leq K_{\mu}(z) \quad \text { a.e. }
$$

Thus, $K_{\mu}^{T}\left(z, z_{0}\right) \neq 0$ and $\infty$ a.e. if $K_{\mu}(z)$ is locally integrable.

Theorem 8.1. Let $\mu: D \rightarrow \mathbb{C}$ be a measurable function with $|\mu(z)|<1$ a.e. and $K_{\mu} \in L_{\mathrm{loc}}^{1}(D)$. Suppose that every point $z_{0} \in D$ has a neighborhood $U_{z_{0}}$ such that

$$
K_{\mu}^{T}\left(z, z_{0}\right) \leq Q_{z_{0}}(z) \quad \text { a.e. }
$$

for some function $Q_{z_{0}}(z)$ of finite mean oscillation at the point $z_{0}$ in the variable $z$. Then the Beltrami equation (1.1) has a strong ring solution.

As a consequence we arrive at the following theorem.

Theorem 8.2. Let $\mu: D \rightarrow \mathbb{C}$ be a measurable function with $|\mu(z)|<1$ a.e. such that

$$
K_{\mu}(z)=\frac{1+|\mu(z)|}{1-|\mu(z)|} \leq Q(z) \in F M O .
$$

Then the Beltrami equation (1.1) has a strong ring solution. 
Since every strong ring solution is a homeomorphic ACL solution and since every BMO function is in FMO, the theorem strengthens earlier results about the existence of ACL homeomorphic solutions of the Beltrami equation when the conditions involve majorants of the class BMO in the previous papers [78] and [79].

Theorem 8.1 and Corollary 2.1 yield the statement.

Theorem 8.3. Let $\mu: D \rightarrow \mathbb{C}$ be a measurable function with $|\mu(z)|<1$ a.e. and $K_{\mu} \in L_{\mathrm{loc}}^{1}(D)$. Suppose that at every $z_{0} \in D$

$$
\varlimsup_{\varepsilon \rightarrow 0} f_{B\left(z_{0}, \varepsilon\right)} \frac{\left|1-\frac{\overline{z-z_{0}}}{z-z_{0}} \mu(z)\right|^{2}}{1-|\mu(z)|^{2}} d m(z)<\infty .
$$

Then the Beltrami equation (1.1) has a strong ring solution $f_{\mu}$.

Corollary 8.1. If at every point $z_{0} \in D$

$$
\varlimsup_{\varepsilon \rightarrow 0} \underset{B\left(z_{0}, \varepsilon\right)}{f} \frac{1+|\mu(z)|}{1-|\mu(z)|} d m(z)<\infty,
$$

then the Beltrami equation (1.1) has a strong ring solution.

The logarithmic mean of a function $Q$ over the ring $A\left(z_{0}, \varepsilon, \varepsilon_{0}\right)=$ $\left\{z \in \mathbb{C}: \varepsilon<\left|z-z_{0}\right|<\varepsilon_{0}\right\}$ is defined by

$$
M_{\log }^{Q}(\varepsilon)=f_{\varepsilon}^{\varepsilon_{0}} q(t) d \log t:=\frac{1}{\log \varepsilon_{0} / \varepsilon} \int_{\varepsilon}^{\varepsilon_{0}} q(t) \frac{d t}{t}
$$

where $q(t)$ denotes the mean value of $Q$ over the circle $\left|z-z_{0}\right|=t$. The following statement is formulated in terms of the logarithmic mean of the tangential dilatation $K_{\mu}^{T}\left(z, z_{0}\right)$ for a fixed $\varepsilon_{0}=\delta\left(z_{0}\right)<\operatorname{dist}\left(z_{0}, \partial D\right)$.

Theorem 8.4. Let $\mu: D \rightarrow \mathbb{C}$ be a measurable function with $|\mu(z)|<1$ a.e. and $K_{\mu} \in L_{\mathrm{loc}}^{1}$. If at every point $z_{0} \in D$ the logarithmic mean of $K_{\mu}^{T}$ over $A(\varepsilon)$ does not converge to $\infty$ as $\varepsilon \rightarrow 0$, i.e.,

$$
\liminf _{\varepsilon \rightarrow 0} M_{l o g}^{K_{\mu}^{T}}(\varepsilon)<\infty
$$

then the Beltrami equation (1.1) has a strong ring solution. 


\section{Extension of the Lehto existence theorem}

In [57] Lehto studied the degenerate Beltrami equations in a special case where the singular set

$$
S_{\mu}=\left\{z \in \mathbb{C}: \lim _{\varepsilon \rightarrow 0}\left\|K_{\mu}\right\|_{L^{\infty}(B(z, \varepsilon))}=\infty\right\}
$$

of the complex coefficient $\mu$ in the Beltrami equation (1.1) is of measure zero. He showed that, if for every $z_{0} \in \mathbb{C}$ and every $r_{1}$ and $r_{2} \in(0, \infty)$ the integral

$$
\int_{r_{1}}^{r_{2}} \frac{d r}{r\left(1+q_{z_{0}}^{T}(r)\right)}, \quad r_{2}>r_{1}
$$

is positive and tends to $\infty$ as either $r_{1} \rightarrow 0$ or $r_{2} \rightarrow \infty$ where

$$
q_{z_{0}}^{T}(r)=\frac{1}{2 \pi} \int_{0}^{2 \pi} \frac{\left|1-e^{-2 i \vartheta} \mu\left(z_{0}+r e^{i \vartheta}\right)\right|^{2}}{1-\left|\mu\left(z_{0}+r e^{i \vartheta}\right)\right|^{2}} d \vartheta
$$

then there exists a homeomorphism $f: \overline{\mathbb{C}} \rightarrow \overline{\mathbb{C}}$ which is ACL in $\mathbb{C} \backslash S_{\mu}$ and satisfies the Beltrami equation (1.1) a.e. Note that the integrand in (9.3) is just the tangential dilatation $K_{\mu}^{T}\left(z, z_{0}\right)$.

We present now a generalization and strengthening of the Lehto existence theorem which enables to derive a series of other existence theorems some of which are known and some are new. This extended version of the Lehto theorem states the existence of a strong ring solution in a domain $D \subset \mathbb{C}$ which by the definition is ACL in $D$ and not only in $D \backslash S_{\mu}$. Note that the situation where $S_{\mu}=D$ is possible here and that the condition (9.4) is weaker than the Lehto condition (9.2) because $q_{z_{0}}^{T}(r)$ can be arbitrarily close to 0 . See Remark 9.1 further for the case where $\infty \in D$, too.

Theorem 9.1. Let $D$ be a domain in $\mathbb{C}$ and let $\mu: D \rightarrow \mathbb{C}$ be a measurable function with $|\mu(z)|<1$ a.e. and $K_{\mu} \in L_{\text {loc }}^{1}(D)$. Suppose that at every point $z_{0} \in D$

$$
\int_{0}^{\delta\left(z_{0}\right)} \frac{d r}{r q_{z_{0}}^{T}(r)}=\infty
$$

where $\delta\left(z_{0}\right)<\operatorname{dist}\left(z_{0}, \partial D\right)$ and $q_{z_{0}}^{T}(r)$ is the mean of $K_{\mu}^{T}\left(z, z_{0}\right)$ over $\left|z-z_{0}\right|=r$. Then the Beltrami equation (1.1) has a strong ring solution $f_{\mu}$. 
A version of this theorem for the ring solutions was first published in the preprint [80], and then in [81], see also [63]. The advanced version for the strong ring solutions was publised in [85]. Note that the centers $z_{0}$ are assumed to belong to the domain $D$ in the case of a ring solution. In the case of strong ring solutions $z_{0}$ can belong to the boundary of $D$.

Note that already in the work [67] it has been established the existence of homeomorphic solutions to the Beltrami equation (1.1) in the class $f_{\mu} \in W_{\mathrm{loc}}^{1, s}, s=2 p /(1+p)$, under the condition (9.4) with $K_{\mu} \in L_{\mathrm{loc}}^{p}$, $p>1$, instead of $K_{\mu}^{T}\left(z, z_{0}\right)$ (the case $p=1$ is covered thanking to a new convergence theorem in Section 3). The Miklyukov-Suvorov result was again discovered in the paper [21] whose author thanks Professor F. W. Gehring but does not mention [67]. Perhaps, the work [67] remains unknown even for the leading experts in the west because we have found no reference to this work in the latest monograph on the Beltrami equations under the discussion of the Lehto condition, see Theorem 20.9.4 in [5].

Theorem 9.1, side by side with lemmas in Section 4, is the main tool for deriving theorems on existence of strong ring solutions for Beltrami equations with various integral conditions in the next section.

Corollary 9.1. If $K_{\mu} \in L_{\mathrm{loc}}^{1}(D)$ and at every point $z_{0} \in D$

$$
q_{z_{0}}^{T}(r)=O\left(\log \frac{1}{r}\right) \quad \text { as } r \rightarrow 0,
$$

then the Beltrami equation (1.1) has a strong ring solution.

Since $K_{\mu}^{T}\left(z, z_{0}\right) \leq K_{\mu}(z)$ we obtain as a consequence of Theorem 9.1 the following result which is due to Miklyukov and Suvorov [67] for the case $K_{\mu} \in L_{\text {loc }}^{p}(D), p>1$.

Corollary 9.2. If $K_{\mu} \in L_{\mathrm{loc}}^{p}(D)$ for $p \geq 1$ and (9.4) holds for $K_{\mu}(z)$ instead of $K_{\mu}^{T}\left(z, z_{0}\right)$ for every point $z_{0} \in D$, then the Beltrami equation (1.1) has a $W_{\mathrm{loc}}^{1, s}(D)$ homeomorphic solution where $s=2 p /(p+1)$.

Remark 9.1. A strong ring solution of the Beltrami equation (1.1) belongs just to the class $W_{\text {loc }}^{1, s}(D)$ where $s=2 p /(p+1)$ if $K_{\mu} \in L_{\text {loc }}^{p}(D)$ for $p \geq 1$.

All the above theorems can be extended to the case where $\infty \in D \subset \overline{\mathbb{C}}$ if one replaces $(9.4)$ by the following condition at $\infty$

$$
\int_{\delta}^{\infty} \frac{d r}{r q(r)}=\infty
$$


where $\delta>0$ and

$$
q(r)=\frac{1}{2 \pi} \int_{0}^{2 \pi} \frac{\left|1-e^{-2 i \vartheta} \mu\left(r e^{i \vartheta}\right)\right|^{2}}{1-\left|\mu\left(r e^{i \vartheta}\right)\right|^{2}} d \vartheta .
$$

In this case, there exists a homeomorphic $W_{\text {loc }}^{1,1}(D)$ solution $f=f_{\mu}$ in $D$ with $f(\infty)=\infty$ and $f_{\mu}^{-1} \in W_{\text {loc }}^{1,2}(f(D))$. Here $f \in W_{\text {loc }}^{1,1}(D)$ in $D$ means that $f \in W_{\text {loc }}^{1,1}(D \backslash\{\infty\})$ and that $f^{*}(z)=1 / \overline{f(1 / \bar{z})}$ belongs to $W^{1,1}$ in a neighborhood of 0 . The statement $f^{-1} \in W_{\text {loc }}^{1,2}(f(D))$ has a similar meaning.

\section{Exixtence theorems with integral conditions}

The following existence theorem is obtained from Theorems 4.2 and 9.1 .

Theorem 10.1. Let $\mu: D \rightarrow \mathbb{C}$ be a measurable function with $|\mu(z)|<1$ a.e. and $K_{\mu} \in L_{\text {loc }}^{1}$. Suppose that every point $z_{0} \in D$ has a neighborhood $U_{z_{0}}$ where

$$
\int_{U_{z_{0}}} \Phi_{z_{0}}\left(K_{\mu}^{T}\left(z, z_{0}\right)\right) d m(z)<\infty
$$

for a non-decreasing convex function $\Phi_{z_{0}}:[0, \infty) \rightarrow[0, \infty]$ such that

$$
\int_{\Delta\left(z_{0}\right)}^{\infty} \frac{d \tau}{\tau \Phi_{z_{0}}^{-1}(\tau)}=\infty
$$

for some $\Delta\left(z_{0}\right)>\Phi_{z_{0}}(0)$. Then the Beltrami equation (1.1) has a strong ring solution.

Remark 10.1. Note that the additional condition $\Delta\left(z_{0}\right)>\Phi_{z_{0}}(0)$ is essential, see also Remark 4.3. In fact, it is important only degree of convergence $\Phi_{z_{0}}^{-1}(\tau) \rightarrow \infty$ as $\tau \rightarrow \infty$ or, the same, degree of convergence $\Phi_{z_{0}}(t) \rightarrow \infty$ as $t \rightarrow \infty$.

Corollary 10.1. Let $\mu: D \rightarrow \mathbb{C}$ be a measurable function with $|\mu(z)|<1$ a.e. and $K_{\mu} \in L_{\mathrm{loc}}^{1}$. Suppose that

$$
\int_{D} \Phi\left(K_{\mu}(z)\right) d m(z)<\infty
$$


for a non-decreasing convex function $\Phi:[0, \infty] \rightarrow[0, \infty]$ such that

$$
\int_{\Delta}^{\infty} \frac{d \tau}{\tau \Phi^{-1}(\tau)}=\infty
$$

for some $\Delta>\Phi(0)$. Then the Beltrami equation (1.1) has a strong ring solution.

In particular, the latter means that under the conditions (10.3) and (10.4) there exists a homeomorphic solution $f$ of (1.1) in the Sobolev class $W_{\text {loc }}^{1,1}$ with the Jacobian $J_{f}(z) \neq 0$ a.e.

Some new criteria of the existence of strong ring solutions for the Beltrami equation (1.1) which will be given further follow from Theorems 4.1 and 10.1 .

Corollary 10.2. Let $\mu: D \rightarrow \mathbb{C}$ be a measurable function with $|\mu(z)|<1$ a.e. and $K_{\mu} \in L_{\mathrm{loc}}^{1}$. If the condition (10.1) holds at every point $z_{0} \in D$ with a non-decreasing convex function $\Phi_{z_{0}}:[0, \infty) \rightarrow[0, \infty)$ such that

$$
\int_{\Delta\left(z_{0}\right)}^{\infty} \log \Phi_{z_{0}}(t) \frac{d t}{t^{2}}=\infty
$$

for some $\Delta\left(z_{0}\right)>0$, then the Beltrami equation (1.1) has a strong ring solution.

Corollary 10.3. Let $\mu: D \rightarrow \mathbb{C}$ be a measurable function with $|\mu(z)|<1$ a.e. and $K_{\mu} \in L_{\mathrm{loc}}^{1}$. If the condition (10.1) holds at every point $z_{0} \in D$ for a continuous non-decreasing convex function $\Phi_{z_{0}}:[0, \infty) \rightarrow[0, \infty)$ such that

$$
\int_{\Delta\left(z_{0}\right)}^{\infty}\left(\log \Phi_{z_{0}}(t)\right)^{\prime} \frac{d t}{t}=\infty
$$

for some $\Delta\left(z_{0}\right)>0$, then (1.1) has a strong ring solution.

Corollary 10.4. Let $\mu: D \rightarrow \mathbb{C}$ be a measurable function with $|\mu(z)|<1$ a.e. and $K_{\mu} \in L_{\text {loc }}^{1}$. If the condition (10.1) holds at every point $z_{0} \in D$ for $\Phi_{z_{0}}=\exp H_{z_{0}}$ where $H_{z_{0}}$ is non-constant, non-decreasing and convex, then (1.1) has a strong ring solution.

Corollary 10.5. Let $\mu: D \rightarrow \mathbb{C}$ be a measurable function with $|\mu(z)|<1$ a.e. and $K_{\mu} \in L_{\mathrm{loc}}^{1}$. If the condition (10.1) holds at every point $z_{0} \in$ 
$D$ for $\Phi_{z_{0}}=\exp H_{z_{0}}$ with a twice continuously differentiable increasing function $H_{z_{0}}$ such that

$$
\left.H_{z_{0}}^{\prime \prime}(t)\right) \geq 0 \quad \forall t \geq t\left(z_{0}\right) \in[0, \infty),
$$

then (1.1) has a strong ring solution.

Theorem 10.2. Let $\mu: D \rightarrow \mathbb{C}$ be a measurable function with $|\mu(z)|<1$ a.e. and $K_{\mu} \in L_{\mathrm{loc}}^{1}$ such that

$$
\int_{D} \Phi\left(K_{\mu}(z)\right) d m(z)<\infty
$$

where $\Phi:[0, \infty) \rightarrow[0, \infty]$ is non-decreasing and convex such that

$$
\int_{\Delta}^{\infty} \log \Phi(t) \frac{d t}{t^{2}}=\infty
$$

for some $\Delta>0$. Then the Beltrami equation (1.1) has a strong ring solution.

Corollary 10.6. Let $\mu: D \rightarrow \mathbb{C}$ be a measurable function with $|\mu(z)|<1$ a.e. and $K_{\mu} \in L_{\mathrm{loc}}^{1}$. If the condition (10.8) holds with a non-decreasing convex function $\Phi:[0, \infty) \rightarrow[0, \infty)$ such that

$$
\int_{t_{0}}^{\infty}(\log \Phi(t))^{\prime} \frac{d t}{t}=\infty
$$

for some $t_{0}>0$, then (1.1) has a strong ring solution.

Corollary 10.7. Let $\mu: D \rightarrow \mathbb{C}$ be a measurable function with $|\mu(z)|<1$ a.e. If the condition (10.8) holds for $\Phi=e^{H}$ where $H$ is non-constant, non-decreasing and convex, then (1.1) has a strong ring solution.

Corollary 10.8. Let $\mu: D \rightarrow \mathbb{C}$ be a measurable function with $|\mu(z)|<1$ a.e. If the condition (10.8) holds for $\Phi=e^{H}$ where $H$ is twice continuously differentiable, increasing and

$$
H^{\prime \prime}(t) \geq 0 \quad \forall t \geq t_{0} \in[1, \infty),
$$

then (1.1) has a strong ring solution. 
Note that among twice continuously differentiable functions, the condition (10.11) is equivalent to the convexity of $H(t), t \geq t_{0}$, cf. Corollary 10.7. Of course, the convexity of $H(t)$ implies the convexity of $\Phi(t)=e^{H(t)}, t \geq t_{0}$, because the function $\exp x$ is convex. However, in general, the convexity of $\Phi$ does not imply the convexity of $H(t)=\log \Phi(t)$ and it is known that the convexity of $\Phi(t)$ in (10.8) is not sufficient for the existence of ACL homeomorphic solutions of the Beltrami equation. There exist examples of the complex coefficients $\mu$ such that $K_{\mu} \in L^{p}$ with an arbitrarily large $p \geq 1$ for which the Beltrami equation (1.1) has no ACL homeomorphic solutions, see e.g. [78].

Remark 10.2. The integral condition (10.1) is replaced at $\infty$ by the following condition

$$
\int_{|z|>\delta} \Phi_{\infty}\left(K_{\mu}^{T}(z, \infty)\right) \frac{d m(z)}{|z|^{4}}<\infty
$$

where $\delta>0, \Phi_{\infty}$ satisfies the conditions of either Theorem 10.1 or equivalent conditions from Theorem 4.1 and

$$
K_{\mu}^{T}(z, \infty)=\frac{\left|1-\frac{\bar{z}}{z} \mu(z)\right|^{2}}{1-|\mu(z)|^{2}} .
$$

We may assume in all the above theorems that the functions $\Phi_{z_{0}}(t)$ and $\Phi(t)$ are not convex on the whole segments $[0, \infty]$ and $[1, \infty]$, respectively, but only on a segment $[T, \infty]$ for some $T \in(1, \infty)$. Indeed, every non-decreasing function $\Phi:[1, \infty] \rightarrow[0, \infty]$ which is convex on a segment $[T, \infty], T \in(0, \infty)$, can be replaced by a non-decreasing convex function $\Phi_{T}:[0, \infty] \rightarrow[0, \infty]$ in the way described in Section 5 .

\section{Necessity of integral conditions for solvability}

The main idea for the proof of the following statement under smooth increasing functions $\Phi$ with the additional condition that $t(\log \Phi)^{\prime} \geq 1$ is due to Iwaniec and Martin, see Theorem 3.1 in [46], cf. also Theorem 11.2.1 in [45] and Theorem 20.3.1 in [5]. We obtain the same conclusion in Lemma 11.1 and Theorem 11.1 further without these smooth conditions. Moreover, by Theorem 4.1 the same conclusion concerns all conditions (4.5)-(4.9).

Theorem 11.1. Let $\Phi:[0, \infty] \rightarrow[0, \infty]$ be such a non-decreasing convex function that, for every measurable function $\mu: \mathbb{D} \rightarrow \mathbb{D}$ satisfying the condition

$$
\int_{\mathbb{D}} \Phi\left(K_{\mu}(z)\right) d m(z)<\infty
$$


the Beltrami equation (1.1) has a homeomorphic ACL solution. Then there is $\delta>0$ such that

$$
\int_{\delta}^{\infty} \log \Phi(t) \frac{d t}{t^{2}}=\infty .
$$

It is evident that the function $\Phi(t)$ in Theorem 11.1 is not constant on $[0, \infty)$ because in the contrary case we would have no real restrictions for $K_{\mu}$ from (11.1) except $\Phi(t) \equiv \infty$ when the class of such $\mu$ is empty. Moreover, by the well-known criterion of convexity, see e.g. Proposition 5 in I.4.3 of [17], the slope $\Phi(t) / t$ is nondecreasing. Hence the proof of Theorem 11.1 is reduced to the following statement.

Lemma 11.1. Let a function $\Phi:[0, \infty] \rightarrow[0, \infty]$ be non-decreasing and

$$
\Phi(t) \geq C \cdot t \quad \forall t \geq T
$$

for some $C>0$ and $T \in(1, \infty)$. If the Beltrami equations (1.1) have $A C L$ homeomorphic solutions for all measurable functions $\mu: \mathbb{D} \rightarrow \mathbb{D}$ satisfying the condition (11.1), then (11.2) holds for some $\delta>0$.

Remark 11.1. Note that the Iwaniec-Martin condition $t(\log \Phi)^{\prime} \geq 1$ implies (11.3) with $C=\Phi(T) / T$. Note also that if we take $\beta_{n+1}=\varepsilon_{n} \rightarrow$ 0 as $n \rightarrow \infty, \alpha_{n+1}=b_{n} e^{b_{n} \gamma_{n}}-\varepsilon_{n} \gamma_{n}$ and $\gamma_{n+1}^{*}=b_{n} e^{b_{n} \gamma_{n}} / \varepsilon_{n}$, in the construction of examples to Lemma 13.1, then we get $\Phi\left(\gamma_{n+1}^{*}\right) / \gamma_{n+1}^{*} \leq$ $2 \varepsilon_{n}$. Thus, we arrive at examples of absolutely continuous increasing functions $\Phi$ with $\Phi(t) \rightarrow \infty$ as $t \rightarrow \infty$ satisfying the condition (11.2) and such that

$$
\liminf _{t \rightarrow \infty} \frac{\Phi(t)}{t}=0 .
$$

Now we see that conditions of the type (11.3) are independent on the conditions (4.4)-(4.9).

In view of its special importance, we give here the corresponding example to Lemma 11.1. Let $\Phi$ fail to satisfy (11.2). Then there exists a measurable function $\mu: \mathbb{D} \rightarrow \mathbb{D}$ such that (11.1) holds, however the Beltrami equation (1.1) has no homeomorphic ACZ solution.

Example 11.1. Let us assume that the condition (11.2) does not hold for any $\delta>0$. Set $t_{0}=\sup _{\Phi(t)=0} t, t_{0}=0$ if $\Phi(t)>0$ for all $t \in[0, \infty]$. Then for all $\delta>t_{0}$

$$
\int_{\delta}^{\infty} \log \Phi(t) \frac{d t}{t^{2}}<\infty .
$$


With no loss of generality, applying the linear transformation $\alpha \Phi+\beta$ with $\alpha=1 / C$ and $\beta=T$, we may assume by (11.3) that

$$
\Phi(t) \geq t \quad \forall t \in[0, \infty) .
$$

Of course, we may also assume that $\Phi(t)=t$ for all $t \in[0,1)$ because the values of $\Phi$ in $[0,1)$ give no information on $K_{\mu}$ in (11.1). Finally, by (11.5) we have that $\Phi(t)<\infty$ for every $t \in[0, \infty)$.

Now, note that the function $\Psi(t):=t \Phi(t)$ is strictly increasing, $\Psi(1)=\Phi(1)$ and $\Psi(t) \rightarrow \infty$ as $t \rightarrow \infty, \Psi(t)<\infty$ for every $t \in[0, \infty)$. Hence the functional equation

$$
\Psi(K(r))=\left(\frac{\gamma}{r}\right)^{2} \quad \forall r \in(0,1],
$$

where $\gamma=\Phi^{1 / 2}(1) \geq 1$, is well solvable. Its solution $K:(0,1] \rightarrow[1, \infty)$ is a continuous non-increasing function such that $K(r) \rightarrow \infty$ as $r \rightarrow 0$ and $K(1)=1$. Taking the logarithm in (11.7), we have that

$$
2 \log r+\log K(r)+\log \Phi(K(r))=2 \log \gamma
$$

and by (11.6) we obtain

$$
\log r+\log K(r) \leq \log \gamma,
$$

i.e.,

$$
K(r) \leq \frac{\gamma}{r}
$$

By (11.7)

$$
\Phi(K(r)) \geq \frac{\gamma}{r}
$$

and hence by $(4.2)$

$$
K(r) \geq \Phi^{-1}\left(\frac{\gamma}{r}\right)
$$

Thus,

$$
I(t):=\int_{0}^{t} \frac{d r}{r K(r)} \leq \int_{0}^{t} \frac{d r}{r \Phi^{-1}\left(\frac{\gamma}{r}\right)}=\int_{\frac{\gamma}{t}}^{\infty} \frac{d \tau}{\tau \Phi^{-1}(\tau)}, \quad t \in(0,1],
$$

where $\gamma / t \geq \gamma \geq 1>\Phi(+0)=0$. By the condition (11.5) and Theorem 4.1

$$
I(t) \leq I(1)=\int_{0}^{1} \frac{d r}{r K(r)}<\infty .
$$


Now consider the mapping

$$
f(z)=\frac{z}{|z|} \rho(|z|)
$$

where $\rho(t)=e^{I(t)}$. Note that $f \in C^{1}(\mathbb{D} \backslash\{0\})$ and hence $f$ is locally quasiconformal in the punctured unit disk $\mathbb{D} \backslash\{0\}$ by the continuity of the function $K(r), r \in(0,1)$, see also (11.9). Let us calculate its complex dilatation. Set $z=r e^{i \vartheta}$. Then

$$
\mu(z)=e^{2 i \vartheta} \cdot \frac{1-K(r)}{1+K(r)}=-\frac{z}{\bar{z}} \cdot \frac{K(|z|)-1}{K(|z|)+1} .
$$

Consequently,

$$
K_{\mu}(z)=K(|z|)
$$

and by (11.7)

$$
\int_{\mathbb{D}} \Phi\left(K_{\mu}(z)\right) d m(z)=2 \pi \int_{0}^{1} \Phi(K(r)) r d r \leq 2 \pi \gamma^{2} I(1)<\infty .
$$

However,

$$
\lim _{z \rightarrow 0}|f(z)|=\lim _{t \rightarrow 0} \rho(t)=e^{I(0)}=1,
$$

i.e., $f$ maps the punctured disk $\mathbb{D} \backslash\{0\}$ onto the ring $1<|\zeta|<R=e^{I(1)}$.

Let us assume that there is a homeomorphic ACL solution $g$ of the Beltrami equation (1.1) with the given $\mu$. By the Riemann theorem without loss of generality we may assume that $g(0)=0$ and $g(\mathbb{D})=\mathbb{D}$. Since $f$ as well as $g$ are locally quasiconformal in the punctured disk $\mathbb{D} \backslash\{0\}$, then by the uniqueness theorem for the quasiconformal mappings $f=h \circ g$ in $\mathbb{D} \backslash\{0\}$ where $h$ is a conformal mapping in $\mathbb{D} \backslash\{0\}$. However, isolated singularities are removable for conformal mappings. Hence $h$ can be extended by continuity to 0 and, consequently, $f$ should be so. Thus, the obtained contradiction disproves the assumption (11.5).

Remark 11.2. Theorems 4.1 and 11.1 show that each of the sufficient conditions (4.4)-(4.9) for the solvability of the Beltrami equations (1.1) with the integral constraints (11.1) and non-decreasing convex functions $\Phi$ is also necessary. 


\section{On the Direchlet problem}

Boundary problems for the Beltrami equations (1.1) are due to the famous dissertation of Riemann who considered a particular case of analytical functions when $\mu(z) \equiv 0$, and to the works of Hilbert $(1904,1924)$ who studied the corresponding system of Cauchy-Riemann for the real and imaginary parts of analytic functions $f=u+i v$, as well as to the work of Poincare (1910) on rising tides.

The Dirichlet problem for the uniformly elliptic Beltrami equations was studied by many authors, see, e.g., [10] and [108], Chapter 4, and the references therein. For an approximate method for solving the Dirichlet problem, see [68]. The same problem for two-dimensional quasilinear differential equation of elliptic type was also investigated in a number of papers, see, e.g., [24] and the corresponding references. Our goal here is to show how to apply our new results on the existence of strong ring solutions for the degenerate Beltrami equations to the study of some boundary value problems.

Every analytic function $f$ in a domain $D$ satisfies the simplest Beltrami equation

$$
f_{\bar{z}}=0
$$

when $\mu(z) \equiv 0$. Recall that if an analytic function $f$ defined in the unit disk $\mathbb{D}=\{z \in \mathbb{C}:|z|<1\}$ is continuous in its closure, then by the Schwarz formula

$$
f(z)=i \operatorname{Im} f(0)+\frac{1}{2 \pi i} \int_{|\zeta|=1} \operatorname{Re} f(\zeta) \cdot \frac{\zeta+z}{\zeta-z} \frac{d \zeta}{\zeta},
$$

see e.g. Section 8 , Ch. III, Part 3 in [43], p. 346. Thus, the analytic function $f$ in the unit disk $\mathbb{D}$ is defined by its real part $\varphi(\zeta)=\operatorname{Re} f(\zeta)$ on the boundary of the unit disk up to a purely imaginary number $i c$, $c=\operatorname{Im} f(0)$.

Similarly, the Dirichlet problem for the Beltrami equation (1.1) in a domain $D \subset \mathbb{C}$ is formulated as the problem on the existence of a continuous function $f: D \rightarrow \mathbb{C}$ which has partial derivatives of the first order satisfying (1.1) a.e. in $D$ and such that

$$
\lim _{z \rightarrow \zeta} \operatorname{Re} f(z)=\varphi(\zeta) \quad \forall \zeta \in \partial D
$$

for a prescribed continuous function $\varphi: \partial D \rightarrow \mathbb{R}$. It is obvious that if $f$ is a solution of such problem, then the function $F(z)=f(z)+i c, c \in \mathbb{R}$, is also a solution. 
The regular solution of the Dirichlet problem (12.3) with $\varphi(\zeta) \equiv c$, $\zeta \in \partial D$, for the Beltrami equation (1.1) is the function $f(z) \equiv c, z \in D$. If $\varphi(\zeta) \not \equiv$ const, then the regular solution of such problem is a continuous, discrete and open mapping $f: D \rightarrow \mathbb{C}$ of the Sobolev class $W_{\text {loc }}^{1,1}$ with the Jacobian $J_{f}(z)=\left|f_{z}\right|^{2}-\left|f_{\bar{z}}\right|^{2} \neq 0$ a.e. satisfying the condition (12.3) and (1.1) a.e. If $D=\mathbb{D}$, where $\mathbb{D}$ stands for the open unit disk centered at the origin, we in addition assume that $\operatorname{Im} f(0)=0$.

Recall that a mapping $f: D \rightarrow \mathbb{C}$ is called discrete if the preimage of $f^{-1}(y)$ consists of isolated points for every $y \in \mathbb{C}$ and open if $f$ maps every open set $U \subseteq D$ onto an open set in $\mathbb{C}$.

In what follows, we prove that a regular solution to the Dirichlet problem (12.3) in the unit disc $\mathbb{D}$ exists for every continuous function $\varphi: \partial \mathbb{D} \rightarrow \mathbb{R}$ for wide classes of the degenerate Beltrami equations (1.1). The Dirichlet problem in a bounded simply connected domain $D$ in $\mathbb{C}$ is reduced to the case of the unit disk by the Riemann mapping theorem. Detailed proofs of the following theorems can be found in [29] and [30].

Theorem 12.1. Let $\mu: \mathbb{D} \rightarrow \mathbb{D}$ be a measurable function such that $K_{\mu}$ has the BMO majorant. Then the Beltrami equation (1.1) has a regular solution of the Dirichlet problem (12.3) for every continuous function $\varphi: \partial \mathbb{D} \rightarrow \mathbb{R}$.

Remark 12.1. In particular, we have that if

$$
K_{\mu}(z) \leq Q(z) \in W_{\text {loc }}^{1,2}(\mathbb{D}),
$$

then the conclusion of Theorem 12.1 holds because $W^{1,2}(\mathbb{D}) \subset$ $V M O(\mathbb{D})$, see e.g. [20]. Moreover, in this case $f \in W_{\text {loc }}^{1,2}$, see e.g. [61], and the problem has the unique regular solution for every continuous function $\varphi: \partial \mathbb{D} \rightarrow \mathbb{R}$.

This theorem admits the following generalization.

Theorem 12.2. Let $\mu: \mathbb{D} \rightarrow \mathbb{D}$ be a measurable function such that $K_{\mu} \in L^{1}(\mathbb{D})$ and $K_{\mu}^{T}\left(z, z_{0}\right) \leq Q\left(z, z_{0}\right)$ where the function $Q\left(z, z_{0}\right)$ belongs to $F M O\left(z_{0}\right)$ in $z$ for all $z_{0} \in \overline{\mathbb{D}}$. Then the Beltrami equation (1.1) has a regular solution of the Dirichlet problem (12.3) for every continuous function $\varphi: \partial \mathbb{D} \rightarrow \mathbb{R}$.

Corollary 12.1. Let $\mu: \mathbb{D} \rightarrow \mathbb{D}$ be a measurable function such that

$$
K_{\mu}(z)=\frac{1+|\mu(z)|}{1-|\mu(z)|} \leq Q(z) \in F M O(\overline{\mathbb{D}}) .
$$

Then the Beltrami equation (1.1) has a regular solution of the problem Dirichlet (12.3) for every continuous function $\varphi: \partial \mathbb{D} \rightarrow \mathbb{R}$. 
Corollary 12.2. Let $\mu: \mathbb{D} \rightarrow \mathbb{D}$ be a measurable function with $K_{\mu} \in$ $L^{1}(\mathbb{D})$ such that

$$
\varlimsup_{\varepsilon \rightarrow 0} f_{\mathbb{D}\left(z_{0}, \varepsilon\right)} \frac{\left|1-\frac{\overline{z-z_{0}}}{z-z_{0}} \mu(z)\right|^{2}}{1-|\mu(z)|^{2}} d m(z)<\infty \quad \forall z_{0} \in \overline{\mathbb{D}}
$$

where $\mathbb{D}\left(z_{0}, \varepsilon\right)=\mathbb{D} \cap B\left(z_{0}, \varepsilon\right), B\left(z_{0}, \varepsilon\right)=\left\{z \in \mathbb{C}:\left|z-z_{0}\right|<\varepsilon\right\}$. Then the Beltrami equation (1.1) has a regular solution of the Dirichlet problem (12.3) for every continuous function $\varphi: \partial \mathbb{D} \rightarrow \mathbb{R}$.

Corollary 12.3. Let $\mu: \mathbb{D} \rightarrow \mathbb{D}$ be a measurable function such that

$$
k_{z_{0}}(\varepsilon)=O\left(\log \frac{1}{\varepsilon}\right) \quad \forall z_{0} \in \overline{\mathbb{D}}
$$

as $\varepsilon \rightarrow 0$, where $k_{z_{0}}(\varepsilon)$ is the average of the function $K_{\mu}^{T}\left(z, z_{0}\right)$ over $\mathbb{D} \cap S\left(z_{0}, \varepsilon\right), S\left(z_{0}, \varepsilon\right)=\left\{z \in \mathbb{C}:\left|z-z_{0}\right|=\varepsilon\right\}$. Then the Beltrami equation (1.1) has a regular solution of the Dirichlet problem (12.3) for every continuous function $\varphi: \mathbb{D} \rightarrow \mathbb{R}$.

Remark 12.2. In particular, the conclusion holds if

$$
K_{\mu}^{T}\left(z, z_{0}\right)=O\left(\log \frac{1}{\left|z-z_{0}\right|}\right) \quad \text { as } z \rightarrow z_{0} \forall z_{0} \in \overline{\mathbb{D}} .
$$

Theorem 12.3. Let $\mu: \mathbb{D} \rightarrow \mathbb{D}$ be a measurable function with $K_{\mu} \in$ $L^{1}(\mathbb{D})$ such that

$$
\int_{0}^{\delta\left(z_{0}\right)} \frac{d r}{\left\|K_{\mu}^{T}\right\|_{1}(r)}=\infty \quad \forall z_{0} \in \overline{\mathbb{D}}
$$

where $\left\|K_{\mu}^{T}\right\|_{1}(r)=\int_{\gamma_{r}} K_{\mu}^{T}\left(z, z_{0}\right)|d z|$ is the $L^{1}$ norm of the function $K_{\mu}^{T}\left(z, z_{0}\right)$ over the arcs $\gamma_{r}=\mathbb{D} \cap S\left(z_{0}, r\right)$ of the circles $S\left(z_{0}, r\right)=\{z \in$ $\left.\mathbb{C}:\left|z-z_{0}\right|=r\right\}, 0<\delta\left(z_{0}\right)<1$. Then the Beltrami equation (1.1) has a regular solution of the Dirichlet problem (12.3) for every continuous function $\varphi: \partial \mathbb{D} \rightarrow \mathbb{R}$.

Remark 12.3. Of course, all the above results on the existence of regular solutions to the Dirichlet problem for the Beltrami equation (1.1) can be formulated in terms of the dilatation $K_{\mu}(z)$ because of the estimate $K_{\mu}^{T}\left(z, z_{0}\right) \leq K_{\mu}(z)$.

On the base of Corollary 4.1 we obtain the following consequence of Theorem 12.3. 
Theorem 12.4. Let $\mu: \mathbb{D} \rightarrow \mathbb{D}$ be a measurable function with $K_{\mu} \in$ $L^{1}(\mathbb{D})$ and such that

$$
\int_{D\left(z_{0}\right)} \Phi_{z_{0}}\left(K_{\mu}^{T}\left(z, z_{0}\right)\right) d m(z)<\infty \quad \forall z_{0} \in \overline{\mathbb{D}}
$$

where $D\left(z_{0}\right)=\left\{z \in \mathbb{D}:\left|z-z_{0}\right|<\delta\left(z_{0}\right)\right\}, 0<\delta\left(z_{0}\right)<1$ and $\Phi_{z_{0}}$ : $[0, \infty] \rightarrow[0, \infty]$ is a non-decreasing convex function that satisfies at least one of the conditions (4.4)-(4.9).

Then the Beltrami equation (1.1) has a regular solution of the Dirichlet problem (12.3) for every continuous function $\varphi: \partial \mathbb{D} \rightarrow \mathbb{R}$.

Next, since $K_{\mu}^{T}\left(z, z_{0}\right) \leq K_{\mu}(z)$, Theorem 12.4 implies the following simple but very important consequence.

Corollary 12.4. Let $\mu: \mathbb{D} \rightarrow \mathbb{D}$ be a measurable function such that

$$
\int_{\mathbb{D}} \Phi\left(K_{\mu}(z)\right) d m(z)<\infty
$$

where $\Phi:[0, \infty] \rightarrow[0, \infty]$ is a non-decreasing convex function that satisfies at least one of the conditions (4.4)-(4.9).

Then the Beltrami equation (1.1) has a regular solution of the Dirichlet problem (12.3) for every continuous function $\varphi: \partial \mathbb{D} \rightarrow \mathbb{R}$.

Remark 12.4. Note that the conditions (4.4)-(4.9) are not only sufficient but also necessary to have a regular solution of the Dirichlet problem (12.3) (under every continuous non-constant function $\varphi: \partial \mathbb{D} \rightarrow \mathbb{R}$ ) for all Beltrami equations (1.1) with the integral constraints (12.11). Indeed, by the Stoilow factorization, see e.g. [96] or [97], such a regular solution $f$ should have the representation $f=h \circ g$ where $h$ is a conformal mapping and $g$ is a homeomorphic solution of the class $W_{\text {loc }}^{1,1}(\mathbb{D})$ to the Beltrami equation (1.1). However, the given conditions are necessary by Theorem 11.1 for the existence of such solutions to all Beltrami equations (1.1) with the integral constraints (12.11).

Example 12.1. Let

$$
\mu(z)=k(|z|) \frac{z}{\bar{z}}
$$

where $k(\tau): \mathbb{R} \rightarrow(-1,1)$ is a measurable function such that

$$
\int_{\varepsilon}^{1} \frac{1+k(\tau)}{1-k(\tau)} \frac{d \tau}{\tau}<\infty \quad \forall \varepsilon \in(0,1)
$$


and

$$
\int_{0}^{1} \frac{1+k(\tau)}{1-k(\tau)} \frac{d \tau}{\tau}=\infty
$$

Then a regular solution to the Dirichlet problem (12.3) for the Beltrami equation (1.1) can be written in the explicit form

$$
f(z)=\frac{1}{2 \pi i} \int_{|\zeta|=1} \operatorname{Re} \varphi(\zeta) \cdot \frac{\zeta+\omega(z)}{\zeta-\omega(z)} \cdot \frac{d \zeta}{\zeta}
$$

where

$$
\omega(z)=\frac{z}{|z|} \exp \left(-\int_{|z|}^{1} \frac{1+k(\tau)}{1-k(\tau)} \frac{d \tau}{\tau}\right)
$$

is a regular homeomorphic solution of the Beltrami equation (1.1) with $\mu$ from (12.12), see Propositions 6.4 and 6.5 in [79], cf. [40].

Example 12.2. The example of a complex dilatation $\mu$ which will be given in (12.18) shows that the Dirichlet problem (12.3) for the Beltrami equation (1.1) has no regular solution for every $\varphi \neq$ const even in the case of $\mu \in C^{\infty}(\overline{\mathbb{D}} \backslash\{0\}), K_{\mu} \in L_{\text {loc }}^{\infty}(\overline{\mathbb{D}} \backslash\{0\})$ and $K_{\mu} \in L^{p}(\mathbb{D})$ for any arbitrarily large $p \geq 1$. Moreover, $K_{\mu}$ has only one singularity at zero and no one on the boundary $\partial \mathbb{D}$.

In the monograph [63], it was given the following simple example of the mapping

$$
g(z)=\frac{z}{|z|}\left(1+|z|^{\alpha}\right)=e^{i \vartheta}\left(1+r^{\alpha}\right),
$$

$z=x+i y=r e^{i \vartheta}, \alpha \in(0,1)$, that maps the punctured unit disk $\mathbb{D}_{0}=$ $\mathbb{D} \backslash\{0\}=\{z \in \mathbb{C}: 0<|z|<1\}$ onto the ring $A(0,1,2)=\{z \in \mathbb{C}: 1<$ $|z|<2\}$ and, thus, $g$ has the unremovable singularity at zero.

The complex dilatation of the mapping $g$ is given by

$$
\mu(z)=\frac{g_{\bar{z}}}{g_{z}}=-e^{2 i \vartheta} \cdot \frac{1+(1-\alpha) r^{\alpha}}{1+(1+\alpha) r^{\alpha}} .
$$

If $\alpha \in(0,1)$, then the dilatation

$$
K_{\mu}(z)=\frac{1+|\mu(z)|}{1-|\mu(z)|}=\frac{1+r^{\alpha}}{\alpha r^{\alpha}}=\frac{1}{\alpha}\left(1+r^{-\alpha}\right) .
$$

The dilatation function has only one singularity as $r \rightarrow 0$ and, moreover, $K_{\mu} \in L^{p}(\mathbb{D})$ for every $p<2 / \alpha$. If $\alpha \rightarrow 0$, we see that the degree of integrability of $K_{\mu}$ can be arbitrarily large. Moreover, $K_{\mu} \in L_{\text {loc }}^{\infty}\left(\mathbb{D}_{0}\right)$ 
and $\mu \in C^{\infty}(\overline{\mathbb{D}} \backslash\{0\})$, i.e., $g$ is locally quasiconformal in the punctured $\operatorname{disk} \mathbb{D}_{0}$.

It is easy to show that the Dirichlet problem (12.3) for (1.1) with $\mu$ of the form (12.18) has no regular solutions $f$ with any continuous function $\varphi \neq$ const. Indeed, by the Stoilow factorization theorem $f=A \circ h$ where $A$ is an analytic function and $h$ is a homeomorphism. However, $h$ is a locally quasiconformal mapping outside of some discrete set of points $M$ in $\mathbb{D}_{0}$, because branch points of the analytic function $A$ are isolated, and satisfies the Beltrami the Beltrami equation (1.1) a.e. Thus, by the uniqueness theorem for quasiconformal mappings we have that $\left.h\right|_{\mathbb{D}_{0} \backslash M}=$ $\left.\psi \circ g\right|_{\mathbb{D}_{0} \backslash M}$, where $\psi$ is a conformal mapping in $g\left(\mathbb{D}_{0} \backslash M\right)=g\left(\mathbb{D}_{0}\right) \backslash g(M)$. Note that, since $g$ is a homeomorphism in $\mathbb{D}_{0}$, the set $g(M)$ consists of isolated points and, thus, is removable for the conformal mapping $\psi$. However, then $M$ is removable for $h$ and $h=\psi \circ g$. Consequently, $f=A_{*} \circ g$ where $A_{*}$ is an analytic.

By the definition, the regular solution $f$ is continuous at zero. However, $g^{-1}(w) \rightarrow 0$ as $|w| \rightarrow 1$. Thus $A_{*}(w) \rightarrow f(0)=\operatorname{Re} f(0)$ as $|w| \rightarrow 1$ and by the Riemann-Schwarz symmetry principle, see, e.g., Theorem 3 in $[43$, Part 3, Ch. $4, \Sigma 5], A_{*}$ is extended through the unit circle $|w|=1$. Simultaneously $A_{*}(w) \equiv f(0)$ on this circle and then $A_{*} \equiv$ const, see, e.g., Theorem 2 in [43, Part 3, Ch. 3, Section 2], that is not possible for $\varphi \neq$ const.

Example 12.3. The example of the mapping (12.17) allows to construct an another example of a complex dilatation $\mu$, see (12.19), of the class $C^{\infty}(\mathbb{D}) \backslash\{0\}$ with $K_{\mu} \in L_{\text {loc }}^{\infty}(\mathbb{D})$, for which the Beltrami equation (1.1) has no regular solutions of the Dirichlet problem (12.3) under $\varphi \neq$ const. $K_{\mu}$ has singularities only on $\partial \mathbb{D}$.

Note that the relation (12.17) allows to define the homeomorphism $w=g(z)$ mapping $\mathbb{C}_{*}=\overline{\mathbb{C}} \backslash\{0\}$ onto $\overline{\mathbb{C}} \backslash \overline{\mathbb{D}}, g(\infty)=\infty$; in the polar coordinates : $R=|w|=1+r^{\alpha}, \theta=\arg w=\arg z=\vartheta$. Consider the mapping

$$
G=j \circ g^{-1} \circ j
$$

where $j$ is the inversion with respect to the unit circle in $\mathbb{C}$. By the construction, $\kappa=G(\varsigma)$ maps $\mathbb{D}$ onto $\mathbb{C}$ and $G(\varsigma) \rightarrow \infty$ as $|\varsigma| \rightarrow 1$. In view of the circle symmetry of the mapping $g$,

$$
G=j_{*} \circ g^{-1} \circ j_{*}
$$

where $j_{*}(\varsigma)=1 / \varsigma$ is a conformal mapping.

It is known that

$$
\mu_{g^{-1}}=-\nu_{g} \circ g^{-1}, \quad \nu_{g^{-1}}=-\mu_{g} \circ g^{-1}
$$


where

$$
\nu_{g}(z):=\frac{g_{\bar{z}}}{\overline{g_{z}}}=-e^{2 i \vartheta} \cdot \frac{1+(1-\alpha) r^{\alpha}}{1+(1+\alpha) r^{\alpha}}=\mu_{g}(z),
$$

see, e.g., IC(4) in [1]. In view of the latter, $\mu_{g^{-1}}=\nu_{g^{-1}}$ and then by $\mathrm{IC}(7)$ and $(9)$ in $[1]$

$$
\mu_{G}=-\mu_{g} \circ g^{-1} \circ j_{*}
$$

i.e.

$$
\mu_{G}(\varsigma)=e^{-2 i \gamma} \cdot \frac{1+(1-\alpha)\left[\frac{1}{\rho}-1\right]}{1+(1+\alpha)\left[\frac{1}{\rho}-1\right]}=e^{-2 i \gamma} \cdot \frac{\rho+(1-\alpha)(1-\rho)}{1+(1+\alpha)(1-\rho)}
$$

where $\rho=|\varsigma|$ and $\gamma=\arg \varsigma$. Consequently, we have that

$$
K_{\mu_{G}}(\varsigma)=\frac{1+\left(\frac{1}{\rho}-1\right)}{\alpha\left(\frac{1}{\rho}-1\right)}=\frac{1}{\alpha(1-\rho)}=\frac{1}{\alpha(1-|\varsigma|)} .
$$

Let us show that the Beltrami equation (1.1) has no regular solutions $f$ of the Dirichlet problem (12.3) for $\varphi \neq$ const and $\mu=\mu_{G}$. Assume that there is a regular solution $f$. Then, arguing as in Example 5, we obtain that $f=A \circ G$ in $\mathbb{D}$ where $A$ is an analytic function in $G(\mathbb{D})=\mathbb{C}$. However, the cluster set

$$
C(\partial \mathbb{D}, \operatorname{Re} f)=\left\{w \in \overline{\mathbb{C}}: w=\lim _{n \rightarrow \infty} \operatorname{Re} f\left(z_{n}\right),\left|z_{n}\right| \rightarrow 1\right\}=\varphi(\partial \mathbb{D})
$$

is bounded and $G(\varsigma) \rightarrow \infty$ if and only if $|\varsigma| \rightarrow 1$. We see that $\operatorname{Re} A$ is bounded and $\operatorname{Re} A=$ const by the Liouville theorem for harmonic functions, see, e.g., Theorem 5 in [91, Ch. 5, Section 14]. The latter is not possible for $\varphi \neq$ const.

\section{Historic comments and final remarks}

In this section we give a comparison of our results with earlier results of other authors.

The first investigation of the existence problem for degenerate Beltrami equations with integral constraints (10.8) as in Theorem 10.2 has been made by Pesin [71]. He studied the special case where $\Phi(t)=e^{t^{\alpha}}-1$ with $\alpha>1$. A theorem of Kruglicov [50] is a partial case of Corollary 10.8. Next, David [25] considered the existence problem with measure constraints

$$
\left|\left\{z \in D: K_{\mu}(z)>t\right\}\right| \leq \varphi(t) \quad \forall t \in[1, \infty)
$$

with special $\varphi(t)$ of the form $a \cdot e^{-b t}$ and Tukia [104] with the corresponding constraints in terms of the spherical area. Note that under the 
integral constraints (10.8) of the exponential type $\Phi(t)=\alpha e^{\beta t}, \alpha>0$, the conditions of David and Tukia hold. Thus, the latter results strengthen the Pesin result.

By the well known John-Nirenberg lemma for functions of the class BMO the David conditions are equivalent to the corresponding integral conditions of the exponential type, see e.g. [78] and [79]. More advanced results in terms of FMO can be found in [83] and [85].

The next step has been made by Brakalova and Jenkins [18] who proved the existence of ACL homeomorphic solutions for the case of the integral constraints $(10.1)$ as in Theorem 10.1 with $K_{\mu}(z)$ instead of $K_{\mu}^{T}\left(z, z_{0}\right)$ and with

$$
\Phi_{z_{0}}(t) \equiv \Phi(t)=\exp \left(\frac{\frac{t+1}{2}}{1+\log \frac{t+1}{2}}\right) .
$$

Note that the condition (4.4) in Theorem 4.1 can be easy verified for the function $\Phi(t)$. Indeed,

$$
(\log \Phi(t))^{\prime}=\frac{1}{2} \frac{\log \frac{t+1}{2}}{\left(1+\log \frac{t+1}{2}\right)^{2}} \sim \frac{1}{2} \frac{1}{\log t} \quad \text { as } t \rightarrow \infty .
$$

Moreover, $\Phi^{\prime \prime}(t) \geq 0$ for all $t \geq T$ for large enough $T \in(1, \infty)$ and hence $\Phi$ is convex on the segment [T, $\infty$, see e.g. [17] and Remark 10.2. Thus, the result of Brakalova and Jenkins [18] is a partial case of Corollary 10.3.

Later on, Iwaniec and Martin $[45,46]$ have proven the existence of solutions in the Orlicz-Sobolev classes for the case where

$$
\Phi_{z_{0}}(t) \equiv \Phi(t)=\exp \left(\frac{p t}{1+\log t}\right)
$$

for some $p>0$. Here we see that

$$
(\log \Phi(t))^{\prime}=\frac{p \log t}{(1+\log t)^{2}} \sim \frac{p}{\log t} \quad \text { as } t \rightarrow \infty,
$$

and the convexity condition is also verified, cf. Corollary 10.6. Note that in the both cases (13.2) and (13.4) for each $\lambda>0$

$$
\Phi(t) \geq t^{\lambda} \quad \forall t \geq t_{\lambda} \in[1, \infty) .
$$

We would like to emphasize a remarkable results on the uniqueness and Stoilow factorization for solutions of the Beltrami equations concerning the case

$$
\Phi_{z_{0}}(t) \equiv \Phi(t)=\exp p t
$$


which is due to Astala, Iwaniec, Martin and David [5] and [25]. Note that earlier a theorem on the existence and uniqueness in the class $W_{\text {loc }}^{1,2}$ has been established under the condition $K_{\mu}(z) \leq Q(z) \in W_{\text {loc }}^{1,2}$ in the work [61].

Corollary 10.2 is due to Gutlyanskii, Martio, Sugawa and Vuorinen in [37] and [38] where they have established the existence of ACL homeomorphic solutions of the Beltrami equation (1.1) in $W_{\mathrm{loc}}^{1, s}, s=2 p /(1+p)$, under $K_{\mu} \in L_{\mathrm{loc}}^{p}$ with $p>1$ for

$$
\Phi_{z_{0}}(t) \equiv \Phi(t):=\exp H(t)
$$

with $H(t)$ being a continuous non-decreasing function such that $\Phi(t)$ is convex and

$$
\int_{1}^{\infty} H(t) \frac{d t}{t^{2}}=\infty .
$$

It was one of the most advanced results in the field of criteria for the solvability of the degenerate Beltrami equations because the condition (13.9) is turned out to be not only sufficient but also necessary as it is clear from Theorem 11.1, see also Remark 11.2.

Similar theorems on the existence and uniqueness of solutions in the Orlich-Sobolev classes have been established subsequently in [5] under the condition (13.9) with the smooth $H$ and $t H^{\prime}(t) \geq 5$, see Theorem 20.5.2 therein, cf. Lemma 11.1 and Remark 11.1. However, we have not found the work [38] in the reference list in [5].

Recently Brakalova and Jenkins have proved the existence of ACL homeomorphic solutions under (10.1), again with $K_{\mu}(z)$ instead of $K_{\mu}^{T}\left(z, z_{0}\right)$, and with

$$
\Phi_{z_{0}}(t) \equiv \Phi(t)=h\left(\frac{t+1}{2}\right)
$$

where they assumed that $h$ is increasing and convex and $h(x) \geq C_{\lambda} x^{\lambda}$ for any $\lambda>1$ with some $C_{\lambda}>0$ and

$$
\int_{1}^{\infty} \frac{d \tau}{\tau h^{-1}(\tau)}=\infty
$$

see [19]. Note that the conditions $h(x) \geq C_{\lambda} x^{\lambda}$ for all $\lambda>1$, in particular, under the above sub-exponential integral constraints, see (13.6), imply that $K_{\mu}$ is locally integrable with any degree $p \in[1, \infty)$.

Some of the given conditions are not necessary as it is clear from the results in Section 11 and from the following lemma and remarks. 
Lemma 13.1. There exist continuous increasing convex functions $\Phi$ : $[1, \infty) \rightarrow[1, \infty)$ such that

$$
\begin{aligned}
& \int_{1}^{\infty} \log \Phi(t) \frac{d t}{t^{2}}=\infty \\
& \liminf _{t \rightarrow \infty} \frac{\log \Phi(t)}{\log t}=1
\end{aligned}
$$

and, moreover,

$$
\Phi(t) \geq t \quad \forall t \in[1, \infty) .
$$

Note that the examples from the proof of Lemma 13.1 further can be extended to $[0, \infty]$ by $\Phi(t)=t$ for $t \in[0,1]$ with keeping all the given properties.

Remark 13.1. The condition (13.13) implies, in particular, that there exist no $\lambda>1, C_{\lambda}>0$ and $T_{\lambda} \in[1, \infty)$ such that

$$
\Phi(t) \geq C_{\lambda} \cdot t^{\lambda} \quad \forall t \geq T_{\lambda} .
$$

Thus, in view of Lemma 13.1 and Theorem 10.2, not one in the conditions (13.15) is necessary in the existence theorems for the Beltrami equations with the integral constraints of the type (10.8).

In addition, for the examples of $\Phi$ given in the proof of Lemma 13.1,

$$
\limsup _{t \rightarrow \infty} \frac{\log \Phi(t)}{\log t}=\infty,
$$

cf. Proposition 13.1 further. Finally, all the conditions (4.4)-(4.9) from Theorem 4.1 hold simultaneously with (13.12) because the increasing convex function $\Phi$ is absolutely continuous.

Example 13.1. Further we use the known criterion which says that a function $\Phi$ is convex on an open interval $I$ if and only if $\Phi$ is continuous and its derivative $\Phi^{\prime}$ exists and is non-decreasing in I except a countable set of points in $I$, see e.g. Proposition 1.4.8 in [17]. We construct $\Phi$ by induction sewing together pairs of functions of the two types $\varphi(t)=\alpha+\beta t$ and $\psi(t)=a e^{b t}$ with suitable positive parameters $a, b$ and $\beta$ and possibly negative $\alpha$.

More precisely, set $\Phi(t)=\varphi_{1}(t)$ for $t \in\left[1, \gamma_{1}^{*}\right]$ and $\Phi(t)=\psi_{1}(t)$ for $t \in\left[\gamma_{1}^{*}, \gamma_{1}\right]$ where $\varphi_{1}(t)=t, \gamma_{1}^{*}=e, \psi_{1}(t)=e^{-(e-1)} e^{t}, \gamma_{1}=e+1$. Let us assume that we already constructed $\Phi(t)$ on the segment $\left[1, \gamma_{n}\right]$ and hence that $\Phi(t)=a_{n} e^{b_{n} t}$ on the last subsegment $\left[\gamma_{n}^{*}, \gamma_{n}\right]$ of the 
segment $\left[\gamma_{n-1}, \gamma_{n}\right]$. Then we set $\varphi_{n+1}(t)=\alpha_{n+1}+\beta_{n+1} t$ where the parameters $\alpha_{n+1}$ and $\beta_{n+1}$ are found from the conditions $\varphi_{n+1}\left(\gamma_{n}\right)=\Phi\left(\gamma_{n}\right)$ and $\varphi_{n+1}^{\prime}\left(\gamma_{n}\right) \geq \Phi^{\prime}\left(\gamma_{n}-0\right)$, i.e., $\alpha_{n+1}+\beta_{n+1} \gamma_{n}=a_{n} e^{b_{n} \gamma_{n}}$ and $\beta_{n+1} \geq$ $a_{n} b_{n} e^{b_{n} \gamma_{n}}$. Let $\beta_{n+1}=a_{n} b_{n} e^{b_{n} \gamma_{n}}, \alpha_{n+1}=a_{n} e^{b_{n} \gamma_{n}}\left(1-b_{n} \gamma_{n}\right)$ and choose a large enough $\gamma_{n+1}^{*}>\gamma_{n}$ from the condition

$$
\log \left(\alpha_{n+1}+\beta_{n+1} \gamma_{n+1}^{*}\right) \leq\left(1+\frac{1}{n}\right) \log \gamma_{n+1}^{*}
$$

and, finally, set $\Phi(t) \equiv \varphi_{n+1}(t)$ on $\left[\gamma_{n}, \gamma_{n+1}^{*}\right]$.

Next, we set $\psi_{n+1}(t)=a_{n+1} e^{b_{n+1} t}$ where parameters $a_{n+1}$ and $b_{n+1}$ are found from the conditions that $\psi_{n+1}\left(\gamma_{n+1}^{*}\right)=\varphi_{n+1}\left(\gamma_{n+1}^{*}\right)$ and $\psi_{n+1}^{\prime}\left(\gamma_{n+1}^{*}\right) \geq \varphi_{n+1}^{\prime}\left(\gamma_{n+1}^{*}\right)$, i.e.,

$$
b_{n+1}=\frac{1}{\gamma_{n+1}^{*}} \log \frac{\alpha_{n+1}+\beta_{n+1} \gamma_{n+1}^{*}}{a_{n+1}}
$$

and, taking into account (13.18),

$$
b_{n+1} \geq \frac{\beta_{n+1}}{\alpha_{n+1}+\beta_{n+1} \gamma_{n+1}^{*}} .
$$

Note that (13.19) holds if we take small enough $a_{n+1}>0$ in (13.18). In addition, we may choose here $b_{n+1}>1$.

Now, let us choose a large enough $\gamma_{n+1}$ with $e^{-1} \gamma_{n+1} \geq \gamma_{n+1}^{*}$ from the condition that

$$
\log \psi_{n+1}\left(e^{-1} \gamma_{n+1}\right) \geq e^{-1} \gamma_{n+1}
$$

i.e.,

$$
\log a_{n+1}+b_{n+1} e^{-1} \gamma_{n+1} \geq e^{-1} \gamma_{n+1} .
$$

Note that (13.21) holds for all large enough $\gamma_{n+1}$ because $b_{n+1}>1$ although $\log a_{n+1}$ can be negative.

Setting $\Phi(t)=\psi_{n+1}(t)$ on the segment $\left[\gamma_{n+1}^{*}, \gamma_{n+1}\right]$, we have that

$$
\log \Phi(t) \geq t \quad \forall t \in\left[e^{-1} \gamma_{n+1}, \gamma_{n+1}\right]
$$

where the subsegment $\left[e^{-1} \gamma_{n+1}, \gamma_{n+1}\right] \subseteq\left[\gamma_{n+1}^{*}, \gamma_{n+1}\right]$ has the logarithmic length 1.

Thus, (13.14) holds because by the construction $\Phi(t)$ is absolutely continuous, $\Phi(1)=1$ and $\Phi^{\prime}(t) \geq 1$ for all $t \in[1, \infty)$; the equality (13.12) holds by (13.22); (13.13) by (13.14) and (13.17); (13.16) by (13.22). 
Remark 13.2. Taking in the above construction $\beta_{n+1}=1$ for all $n=1,2, \ldots, \alpha_{n+1}=b_{n} e^{b_{n} \gamma_{n}}-\gamma_{n}$ and arbitrary $\gamma_{n+1}^{*}>\gamma_{n+1}$ we obtain examples of absolutely continuous increasing functions $\Phi$ which are not convex but satisfy (13.12), as well as all the conditions (4.4)-(4.9) from Proposition 4.1, and (13.14).

The corresponding examples of non-decreasing functions $\Phi$ which are neither continuous, nor strictly monotone and nor convex in any neighborhood of $\infty$ but satisfy (13.12), as well as (4.4)-(4.9), and (13.14) are obtained in the above construction if we take $\beta_{n+1}=0$ and $\alpha_{n+1}>\gamma_{n}$ such that $\alpha_{n+1}>\Phi\left(\gamma_{n}\right)$ and $\Phi(t)=\alpha_{n+1}$ for all $t \in\left(\gamma_{n}, \gamma_{n+1}^{*}\right], \gamma_{n+1}^{*}=$ $\alpha_{n+1}$.

Proposition 13.1. Let $\Phi:[1, \infty) \rightarrow[1, \infty)$ be a locally integrable function such that

$$
\int_{1}^{\infty} \log \Phi(t) \frac{d t}{t^{2}}=\infty
$$

Then

$$
\limsup _{t \rightarrow \infty} \frac{\Phi(t)}{t^{\lambda}}=\infty \quad \forall \lambda \in \mathbb{R} .
$$

Remark 13.3. In particular, (13.24) itself implies the relation (13.16). Indeed, we have from (13.24) that there exists a monotone sequence $t_{n} \rightarrow \infty$ as $n \rightarrow \infty$ such that

$$
\Phi\left(t_{n}\right) \geq t_{n}^{n}, \quad n=1,2, \ldots,
$$

i.e.,

$$
\frac{\log \Phi\left(t_{n}\right)}{\log t_{n}} \geq n, \quad n=1,2, \ldots .
$$

Indeed, it is sufficient to consider the case $\lambda>0$. Set $H(t)=\log \Phi(t)$, i.e., $\Phi(t)=e^{H(t)}$. Note that $e^{x} \geq x^{n} / n$ ! for all $x \geq 0$ and $n=1,2 \ldots$, because $e^{x}=\sum_{n=0}^{\infty} x^{n} / n$ !. Fix $\lambda>0$ and $n>\lambda$. Then $q:=\lambda / n$ belongs to $(0,1)$ and

$$
\frac{H(t)}{t^{q}} \leq\left(\frac{\Phi(t)}{t^{\lambda}}\right)^{\frac{1}{n}} \cdot \sqrt[n]{n !} .
$$

Let us assume that

$$
C:=\limsup _{t \rightarrow \infty} \frac{\Phi(t)}{t^{\lambda}}<\infty .
$$

Then

$$
\int_{\triangle}^{\infty} H(t) \frac{d t}{t^{2}}<2 \sqrt[n]{C n !} \int_{\triangle}^{\infty} \frac{d t}{t^{2-q}}=-\left.\frac{2}{1-q} \frac{\sqrt[n]{C n !}}{t^{1-q}}\right|_{\triangle} ^{\infty}=\frac{2}{1-q} \frac{\sqrt[n]{C n !}}{\triangle^{1-q}}<\infty
$$


for large enough $\Delta>1>0$. The latter contradicts (13.23). Hence the assumption (13.27) was not true and, thus, (13.24) holds for all $\lambda \in \mathbb{R}$.

Remark 13.4. Lemma 13.1 shows that, generally speaking, lim sup in (13.24) cannot be replaced by lim for an arbitrary $\lambda>1$ under the condition (13.23) even if $\Phi$ is continuous, increasing and convex.

\section{References}

[1] L. V. Ahlfors, Lectures on Quasiconformal Mappings, D. Van Nostrand Company, Inc., Princeton etc., 1966.

[2] L. Ahlfors, On quasiconformal mappings // J. Anal. Math., 3 (1953/54), 1-58.

[3] L. Ahlfors, A. Beurling, Conformal invariance and function-theoretic null-sets // Acta Math., 83 (1950), 101-129.

[4] C. Andreian Cazacu, On the length-area dilatation // Complex Var., 50 (2005), N 7-11, 765-776.

[5] K. Astala, T. Iwaniec and G. J. Martin, Elliptic differential equations and quasiconformal mappings in the plane, Princeton Math. Ser., v. 48, Princeton Univ. Press, Princeton, 2009.

[6] V. V. Aseev, Moduli of families of locally quasisymmetric surfaces // Sib. Math. J., 30 (1989), N 3, 353-358.

[7] A. K. Bahtin, G. P. Bahtina, Yu. B. Zelinskii, Topologic-algebraic structures and geometric methods in complex analysis, Kiev, Inst. Mat. NAHU, 2008.

[8] P. P. Belinskii, General properties of quasiconformal mappings, Izdat. "Nauka" Sibirsk. Otdel., Novosibirsk, 1974 (Russian).

[9] P. A. Biluta, Extremal problems for mappings quasiconformal in the mean // Sib. Mat. Zh., 6 (1965), 717-726 (Russian).

[10] B. Bojarski, Generalized solutions of a system of differential equations of the first order of the elliptic type with discontinuous coefficients // Mat. Sb., 43(85) (1957), N 4, 451-503 (Russian).

[11] B. Bojarski, Generalized solutions of a system of differential equations of the first order of elliptic type with discontinuous coefficients, Univ. Print. House, Jyväskylä 2009.

[12] B. Bojarski, Subsonic flow of compressible fluid // Arch. Mech. Stoc., 18 (1966), 497-520.

[13] B. Bojarski, Primary solutions of general Beltrami equations // Ann. Acad. Sci. Fenn. Math., 32 (2007), N 2, 549-557.

[14] B. Bojarski, V. Gutlyanskii and V. Ryazanov, General Beltrami equations and BMO // Ukrainian Math. Bull., 5 (2008), N 3, 305-326.

[15] B. Bojarski, V. Gutlyanskii and V. Ryazanov, On the Beltrami equations with two characteristics // Complex Variables and Elliptic Equations, 54 (2009), N 10, 935-950.

[16] B. Bojarski, V. Gutlyanskii and V. Ryazanov, On Integral Conditions for the General Beltrami Equations // Complex Analysis and Operator Theory DOI 10.1007/s11785-010-0088-z. 
[17] N. Bourbaki, Functions of a Real Variable, Springer, Berlin, 2004.

[18] M. A. Brakalova and J. A. Jenkins, On solutions of the Beltrami equation // J. Anal. Math., 76 (1998), 67-92.

[19] M. A. Brakalova and J. A. Jenkins, On solutions of the Beltrami equation. II // Publ. de l'Inst. Math., 75(89) (2004), 3-8.

[20] H. Brezis, L. Nirenberg, Degree theory and BMO. I. Compact manifolds without boundaries // Selecta Math. (N.S.), 1 (1995), N 2, 197-263.

[21] Z. G. Chen, $\mu(z)$-homeomorphisms of the plane // Michigan Math. J., 51 (2003), N 3, 547-556.

[22] F. Chiarenza, M. Frasca and P. Longo, $W^{2, p}$-solvability of the Dirichlet problem for nondivergence elliptic equations with VMO coefficients // Trans. Amer. Math. Soc., 336 (1993), N 2, 841-853.

[23] E. M. Chirka, On the propagation of holomorphic motions // Dokl. Akad. Nauk, 397 (2004), N 1, 37-40.

[24] I. I. Danilyuk, The Dirihlet problem for two-dimensional quasilinear differential equation of elliptic type // Dokl. Akad. Nauk. Ukrain. SSR, Ser A, 12 (1987), $3-7$.

[25] G. David, Solutions de l'equation de Beltrami avec $\|\mu\|_{\infty}=1 / /$ Ann. Acad. Sci. Fenn. Ser. AI. Math. AI., 13 (1988), N 1, 25-70.

[26] V. N. Dubinin, Capacities of condencers and symmetrization in geometric function theory of complex variable, Vladivostok, Dal'nauka, 2009.

[27] J. Dugundji, Topology, Allyn and Bacon Inc., Boston, 1966.

[28] N. Dunford and J. T. Schwartz, Linear Operators, Part I: General Theory, Interscience Publishers, Inc., New York, London, 1957.

[29] Yu. Dybov, The Dirichlet problem for the Beltrami equation // Proceeding of Inst. Appl. Math. Mech. of NAS of Ukraine, 18 (2009), 62-70.

[30] Yu. Dybov, On regular solutions of the Dirichlet problem for the Beltrami equations // Complex Variables and Elliptic Equations (to appear).

[31] L. C. Evans and R. F. Gapiery, Measure Theory and Fine Properties of Functions, CRC Press, Boca Raton, FL, 1992.

[32] F. W. Gehring, Quasiconformal mappings, in Complex Analysis and its Applications, V. 2., International Atomic Energy Agency, Vienna, 1976.

[33] F. W. Gehring, Rings and quasiconformal mappings in space // Trans. Amer. Math. Soc., 103 (1962), 353-393.

[34] F. W. Gehring and O. Lehto, On the total differentiability of functions of a comlex variable // Ann. Acad. Sci. Fenn. Math. AI., 272 (1959), 1-9.

[35] A. Golberg, Homeomorphisms with finite mean dilatations // Contemporary Math., 382 (2005), 177-186.

[36] A. Golberg, V. S. Kud'yavin, Mean coefficients of quasiconformality of pair of domains // Ukrain. Mat. Zh., 43 (1991), N 12, 1709-1712 [in Russian]; translation in Ukrain. Math. J., 43 (1991), 1594-1597.

[37] V. Gutlyanskii, O. Martio, T. Sugawa and M. Vuorinen, On the degenerate Beltrami equation // Reports Dept. Math. Helsinki, 282 (2001), 1-32.

[38] V. Gutlyanskii, O. Martio, T. Sugawa and M. Vuorinen, On the degenerate Beltrami equation // Trans. Amer. Math. Soc., 357 (2005), 875-900. 
[39] V. Gutlyanskii. V. Ryazanov, Quasiconformal mappings with integral constraints on the M. A. Lavrent'ev characteristic // Sibirsk. Mat. Zh., 31 (1990), N 2, 21-36; translation in Siberian Math. J., 31 (1990), N 2, 205-215.

[40] V. Ya. Gutlyanskii, V. I. Ryazanov, On the theory of local behavior for quasiconformal mappings // Izv. Ross. Akad. Nauk. Ser. Math., 59 (1995), N 3, 31-58 (Russian).

[41] J. Heinonen, T. Kilpelainen, O. Martio, Nonlinear Potential Theory of Degenerate Elliptic Equations, Clarendon Press, Oxford Univ. Press, 1993.

[42] S. Hencl and P. Koskela, Regularity of the inverse of a planar Sobolev homeomorphisms, Arch. Ration Mech. Anal., 180 (2006), N 1, 75-95.

[43] A. Hurwitz, R. Courant, The Function theory, Nauka, Moscow, 1968 (Russian).

[44] A. Ignat'ev and V. Ryazanov, Finite mean oscillation in the mapping theory // Ukrainian Math. Bull., 2 (2005), N 3, 403-424.

[45] T. Iwaniec and G. Martin, Geometric Function Theory and Nonlinear Analysis, Clarendon Press, Oxford, 2001.

[46] T. Iwaniec and G. Martin, The Beltrami equation // Memories of AMS, 191 (2008), 1-92.

[47] T. Iwaniec and C. Sbordone, Riesz transforms and elliptic PDEs with VMO coefficients // J. Anal. Math., 74 (1998), 183-212.

[48] F. John and L. Nirenberg, On functions of bounded mean oscillation // Comm. Pure Appl. Math., 14 (1961), 415-426.

[49] L. V. Kovalev, Monotonicity of generalized reduced modulus // Zapiski Nauch. Sem. POMI, 276 (2001), 219-236.

[50] V. I. Kruglikov, The existence and uniqueness of mappings that are quasiconformal in the mean, p. 123-147. In the book: Metric Questions of the Theory of Functions and Mappings, Kiev, Naukova Dumka, 1973 (Russian).

[51] S.L. Krushkal', On mappings quasiconformal in the mean // Dokl. Akad. Nauk SSSR, 157 (1964), N 3, 517-519 (Russian).

[52] S. L. Krushkal', R. Kühnau, Quasiconformal mappings, new methods and applications, Nauka, Novosibirsk, 1984 (Russian); Quasikonfoeme Abbildungenneue Methoden und Anwendungen. Teubner-Text zur Mathematik, Vol. 54 BSB B. G. Teubner Verlagsgesellschaft, Leipzig, 1983 (German).

[53] V.S. Kud'yavin, Local structure of plane mappings quasiconformal in the mean // Doklady Akademii Nauk Ukrainy, 3 (1991), 10-12.

[54] R. Kühnau, Über Extremalprobleme bei im Mittel quasiconformen Abbildungen // Lecture Notes in Math., 1013 (1983), 113-124 (German).

[55] Kuz'mina G.V.: Moduli of the curve families and quadratic differentials, Trudy Mat. Inst. AN SSSR, 139 (1980), 1-240.

[56] M. A. Lavrentieff, On a class of continuous mappings // Mat. Sb., 42 (1935), N 4, 407-424.

[57] O. Lehto, Homeomorphisms with a prescribed dilatation // Lecture Notes in Math., 118 (1968), 58-73.

[58] O. Lehto and K. Virtanen, Quasiconformal Mappings in the Plane, Springer, New York etc., 1973. 
[59] T. Lomako, On extension to the boundary of some generalizations of quasiconformal mappings // Ukrainian Math. J., 61 (2009), 1329-1337.

[60] V. Maz'ya, Sobolev classes, Springer, Berlin-New York, 1985.

[61] O. Martio and V. Miklyukov, On existence and uniqueness of the degenerate Beltrami equation // Complex Variables Theory Appl., 49 (2004), N 7, 647-656.

[62] O. Martio, V. Miklyukov and M. Vuorinen, Some remarks on an existence problem for degenerate elliptic system // Proc. Amer. Math. Soc., 133 (2005), 1451-1458.

[63] O. Martio, V. Ryazanov, U. Srebro and E. Yakubov, Moduli in Modern Mapping Theory, Springer, New York, 2009.

[64] O. Martio, V. Ryazanov and M. Vuorinen, BMO and Injectivity of Space Quasiregular Mappings // Math. Nachr., 205 (1999), 149-161.

[65] V. G. Maz'ya and S. V. Poborchi, Differentiable Functions on Bad Domains, Singapure-New Jersey-London-Hong Kong, World Scientific, 1997.

[66] D. Menchoff, Sur les differentielles totales des fonctions univalentes // Math. Ann., 105 (1931), 75-85.

[67] V. M. Miklyukov and G. D. Suvorov, On existence and uniqueness of quasiconformal mappings with unbounded characteristics, In the book: Investigations in the Theory of Functions of Complex Variables and its Applications, Yu. A. Mitropol'skii, ed., Kiev, Inst. Math., 1972, pp. 45-53.

[68] R. M. Nasyrov, S. R. Nasyrov, Convergence of an approximate method of S. A. Khristianovich for solving the Dirichlet problem for an elliptic equation // Soviet Math. Dokl., 34 (1987), N 3, 484-488.

[69] D. K. Palagachev, Quasilinear elliptic equations with VMO coefficients // Trans. Amer. Math. Soc., 347 (1995), N 7, 2481-2493.

[70] M. Perovich, Isolated singularity of the mean quasiconformal mappings // Lect. Notes in Math., 743 (1979), 212-214.

[71] I. N. Pesin, Mappings quasiconformal in the mean // Dokl. Akad. Nauk SSSR, 187 (1969), N 4, 740-742.

[72] S. P. Ponomarev, The $N^{-1}$-property of mappings, and Lusin's ( $N$ ) condition // Mat. Zametki, 58 (1995), 411-418; transl. in Math. Notes, 58 (1995), 960-965.

[73] M. A. Ragusa, Elliptic boundary value problem in vanishing mean oscillation hypothesis // Comment. Math. Univ. Carolin., 40 (1999), N 4, 651-663.

[74] H. M. Reimann and T. Rychener, Funktionen Beschrankter Mittlerer Oscillation, Springer, Berlin etc., 1975.

[75] Yu. G. Reshetnyak, Space Mappings with Bounded Distortion, Transl. of Math. Monographs 73, AMS, 1989.

[76] V. Ryazanov, On convergence and compactness theorems for ACL homeomorphisms // Rev. Roum. Math. Pures Appl., 41 (1996), 133-139.

[77] V. Ryazanov and R. Salimov, Weakly flat spaces and boudaries in the mapping theory // Ukr. Mat. Vis., 4 (2007), N 2, 199-234 (Russian); translation in Ukrain. Math. Bull., 4 (2007), N 2, 199-233.

[78] V. Ryazanov, U. Srebro and E. Yakubov, BMO-quasiconformal mappings // J. d'Analyse Math., 83 (2001), 1-20.

[79] V. Ryazanov, U. Srebro and E. Yakubov, Plane mappings with dilatation dominated by functions of bounded mean oscillation // Sib. Adv. Math., 11 (2001), N 2, 94-130. 
[80] V. Ryazanov, U. Srebro and E. Yakubov, Degenerate Beltrami equation and radial Q-homeomorphisms // Reports Dept. Math. Helsinki, 369 (2003), 1-34.

[81] V. Ryazanov, U. Srebro and E. Yakubov, On ring solutions of Beltrami equation // J. d'Analyse Math., 96 (2005), 117-150.

[82] V. Ryazanov, U. Srebro and E. Yakubov, Beltrami equation and FMO functions // Contemp. Math., 382 (2005), 357-364.

[83] V. Ryazanov, U. Srebro and E. Yakubov, Finite mean oscillation and the Beltrami equation // Israel J. Math., 153 (2006), 247-266.

[84] V. Ryazanov, U. Srebro and E. Yakubov, On convergence theory for Beltrami equations // Ukrainian Math. Bull., 5 (2008), N 4, 524-535.

[85] V. Ryazanov, U. Srebro and E. Yakubov, On strong solutions of the Beltrami equations // Complex Variables and Elliptic Equations, 55 (2010), N 1-3, 219 236.

[86] V. Ryazanov, U. Srebro and E. Yakubov, Integral conditions in the theory of the Beltrami equations // arXiv: 1001.2821v11 [math.CV] 12 Apr 2010, 1-26.

[87] V. Ryazanov, U. Srebro and E. Yakubov, Integral conditions in the theory of the Beltrami equations // Complex Variables and Elliptic Equations (to appear).

[88] E. Reich and H. Walczak, On the behavior of quasiconformal mappings at a point // Trans. Amer. Math. Soc., 117 (1965), 338-351.

[89] S. Saks, Theory of the Integral, New York, Dover Publ. Inc., 1964.

[90] D. Sarason, Functions of vanishing mean oscillation // Trans. Amer. Math. Soc., 207 (1975), 391-405.

[91] B. V. Shabat, Introduction to Complex Analysis, Nauka, Moscow, 1969, (Russian).

[92] V. A. Shlyk, The equality between p-capacity and p-modulus // Sib. Math. J., 34 (1993), N 6, 1196-1200.

[93] A. Yu. Solynin, Moduli and extremal-metric problems // Algebra and Analysis, 11 (1999), N 1, 3-86.

[94] U. Srebro and E. Yakubov, The Beltrami equation, Handbook in Complex Analysis: Geometric function theory, Vol. 2, 555-597, Elseiver B. V., 2005.

[95] S. L. Sobolev, Applications of functional analysis in mathematical physics, Izdat. Gos. Univ., Leningrad 1950; English transl, Amer. Math. Soc., Providence, R.I., 1963.

[96] S. Stoilow, Lecons sur les Principes Topologue de le Theorie des Fonctions Analytique, Gauthier-Villars, 1938; Riemann, Gauthier-Villars, Paris, 1956 (in French).

[97] S. Stoilow, Lectures on topological principles of the theory of analytic functions, Nauka, Moscow, 1964 (Russian).

[98] Y. F. Strugov, Compactness of the classes of mappings quasiconformal in the mean // Dokl. Akad. Nauk SSSR, 243(4) (1978), 859-861 (Russian).

[99] Y. F. Strugov, A. V. Sychov, On different classes of mappings quasiconformal in the mean // Vest. PANI, 7 (2002), 14-19 (Russian).

[100] G. Suvorov, Families of plane topological mappings, Izdat. Sibirsk. Otdel. Akad. Nauk SSSR, Novosibirsk, 1965 (Russian).

[101] A. V. Sychev, Moduli and spatial quasiconformal mappings, Novosibirsk, Nauka, 1983. 
[102] P. M. Tamrazov, Continuity of certain conformal invariants // Ukrain. Mat. Zh., 18 (1966), N 6, 78-84 (Russian).

[103] Yu. Yu. Trokhimchuk, Differentiation, inner mappings and criteria of analyticity, Kiev, IM NASU, 2008.

[104] P. Tukia, Compactness properties of $\mu$-homeomorphisms // Ann. Acad. Sci. Fenn. Ser. AI. Math. AI., 16 (1991), N 1, 47-69.

[105] A. Uhlov, S. Vodopyanov, Mappings associated with weighted Sobolev spaces // Complex Anal. Dynam. Sys. III. Contemp. Math., 455 (2008), 363-382.

[106] J. Väisälä, Lectures on n-Dimensional Quasiconformal Mappings, Lecture Notes in Math. 229, Berlin etc., Springer-Verlag, 1971.

[107] A. Vasil'ev, Moduli of families of curves for conformal and quasiconformal mappings, Lecture Notes in Math. 1788, Springer-Verlag, Berlin-New York, 2002.

[108] I. N. Vekua, Generalized Analytic Functions, Pergamon Press, 1962.

[109] L. I. Volkovyskiŭ, Quasiconformal mappings, Lvov. Univ., Lvov, 1954 (Russian).

[110] E. Yakubov, Solutions of Beltrami's equation with degeneration // Dokl. Akad. Nauk SSSR, 243 (1978), N 5, 1148-1149.

[111] V. A. Zorich, Quasiconformal mappings and the asymptotic geometry of manifolds // Russ. Math. Surv.,57 (2002), N 3, 437-462; translation from Usp. Mat. Nauk, 57 (2002), N 3, 3-28.

\section{CONTACT INFORMATION}

\section{Vladimir \\ Gutlyanskiü, Vladimir Ryazanov}

\section{Uri Srebro}

Eduard Yakubov
Institute of Applied Mathematics and Mechanics, NAS of Ukraine, ul. Roze Luxemburg 74,

83114, Donetsk,

Ukraine

E-Mail: vladimirgut@mail.ru, vlryazanov1@rambler.ru

Technion - Israel Institute of Technology, Haifa 32000, Israel

E-Mail: srebro@math.technion.ac.il

Holon Institute of Technology,

52 Golomb St., P.O.Box 305,

Holon 58102,

Israel

E-Mail: yakubov@hit.ac.il 